\title{
Cell-mimicking nanodecoys neutralize SARS-CoV-2 and mitigate lung injury in a non-human primate model of COVID-19
}

\author{
Zhenhua Li $\circledast^{1,2,6}$, Zhenzhen Wang ${ }^{1,2,6}$, Phuong-Uyen C. Dinh $\oplus^{1,2,3,6}$, \\ Dashuai Zhu $\oplus^{1,2}$, Kristen D. Popowski $\oplus^{1,2}$, Halle Lutz $\oplus^{1,2}$, Shiqi Hu ${ }^{1,2}$, Mark G. Lewis ${ }^{4}$, Anthony Cook ${ }^{4}$, \\ Hanne Andersen ${ }^{4}$, Jack Greenhouse ${ }^{4}$, Laurent Pessaint ${ }^{4}$, Leonard J. Lobo ${ }^{5}$ and Ke Cheng ${ }^{1,2} \bowtie$
}

Coronavirus disease 2019 (COVID-19), caused by severe acute respiratory syndrome coronavirus 2 (SARS-CoV-2), has grown into a global pandemic, and only a few antiviral treatments have been approved to date. Angiotensin-converting enzyme 2 (ACE2) plays a fundamental role in SARS-CoV-2 pathogenesis because it allows viral entry into host cells. Here we show that ACE2 nanodecoys derived from human lung spheroid cells (LSCs) can bind and neutralize SARS-CoV-2 and protect the host lung cells from infection. In mice, these LSC-nanodecoys were delivered via inhalation therapy and resided in the lungs for over $72 \mathrm{~h}$ post-delivery. Furthermore, inhalation of the LSC-nanodecoys accelerated clearance of SARS-CoV-2 mimics from the lungs, with no observed toxicity. In cynomolgus macaques challenged with live SARS-CoV-2, four doses of these nanodecoys delivered by inhalation promoted viral clearance and reduced lung injury. Our results suggest that LSC-nanodecoys can serve as a potential therapeutic agent for treating COVID-19.

S evere acute respiratory syndrome coronavirus 2 (SARS-CoV-2), the pathogen at the centre of the current global pandemic, causes coronavirus disease 2019 (COVID$19)^{1}$. Coronaviruses are a common type of virus: alpha $(\alpha)$ coronaviruses and beta $(\beta)$ coronaviruses can infect mammals and often manifest as the common cold or gastrointestinal discomfort. Rarely, more severe and lethal forms emerge. SARS-CoV-2 is capable of wreaking havoc on the respiratory and immune system by inducing secretion of proinflammatory cytokines, thereby triggering an increase in alveolar oedema, hypoxaemia, dyspnoea and systemic inflammatory response syndrome ${ }^{2}$. Similar to its deadly predecessors, SARS-CoV-1 (the cause of SARS in 2003) and Middle East respiratory syndrome (MERS)-CoV (the cause of MERS in 2012), SARS-CoV-2 is an enveloped, positive-sense, $\beta$ coronavirus with dangerously high human-to-human transmission rates, with a reported $R_{0}$ (the average number of people that one contagious person will infect) ranging from 2 to 6 (refs. ${ }^{3,4}$ ). Therefore, initial efforts to combat the virus primarily focused on containment to stop the spread and elucidate the pathogenesis of the virus.

It is becoming undeniably evident that in addition to an efficacious vaccine, the development of therapeutics is necessary to completely end this pandemic and provide a solution to treat COVID-19 patients who are severely ill. Researchers around the world are in an urgent race to find an effective therapy for COVID-19. According to the published interim results from the World Health Organization's Solidarity Trial on 15 October 2020, all four of the treatments evaluated to date (remdesivir, hydroxychloroquine, lopinavir/ritonavir and interferon) had little or no effect on overall mortality, necessity for mechanical ventilation and length of hospital stay in hospitalized COVID-19 patients.
Angiotensin-converting enzyme 2 (ACE2), which is present on many cell types and found in almost all tissues, is a carboxypeptidase that plays a pivotal role in host cell viral entry. SARS-CoV-2 specifically attacks ACE2-presenting type II pneumocytes in the lungs and goblet secretory cells in the nasal mucosa by the interaction of its spike protein with ACE2 ${ }^{5}$. In this study, we exploit the virus's cell entry strategy as a Trojan horse to trick the virus. Over the past seven years, our laboratory has developed lung spheroid cells (LSCs) as a cell therapy to treat lung fibrosis and inflammation from initial rodent studies to an ongoing Phase 1 clinical trial $(\mathrm{NCT} 04262167)^{6-9}$. LSCs are a mixture of resident lung epithelial cells (containing both types I and II pneumocytes) and mesenchymal cells. As resident lung cells, they express ACE2, and therefore we fabricated LSC membrane nanovesicles as ACE2 nanodecoys. These LSC-nanodecoys, acting as cell mimics, are capable of binding to the SARS-CoV-2 spike (S) protein and triggering a phagocytic response from macrophages that results in viral elimination.

Therapeutic antibodies and fusion inhibitors have been developed to target the spike protein of SARS-CoV- $2^{10}$. However, more aggressive variants associated with mutations in the spike protein of SARS-CoV-2 have been discovered ${ }^{11,12}$. Therefore, antiviral strategies based on the human receptor ACE2 used by the virus to gain host cell entry, rather than on the viral components, should be of greater interest because no mutations are to be expected on the host cells. Our data support a potential non-invasive therapeutic strategy for neutralizing SARS-CoV-2. Our approach is fundamentally different from the two strategies currently in use, that is, antiviral drugs and vaccines. The LSCs used to fabricate the nanodecoys are generated through a robust, reproducible and scalable culturing method suitable for producing clinically applicable quantities of cell therapy

'Department of Molecular Biomedical Sciences and Comparative Medicine Institute, North Carolina State University, Raleigh, NC, USA. ${ }^{2}$ Joint Department of Biomedical Engineering, The University of North Carolina at Chapel Hill and North Carolina State University, Chapel Hill, NC, USA. ${ }^{3}$ BreStem Therapeutics Inc., Raleigh, NC, USA. " Bioqual Inc., Rockville, MD, USA. ${ }^{5}$ Division of Pulmonary Medicine, The University of North Carolina at Chapel Hill, Chapel Hill, NC, USA. ${ }^{6}$ These authors contributed equally: Z. Li, Z. Wang, P.-U.C. Dinh. ${ }^{凶}$-mail: ke_cheng@ncsu.edu 
a
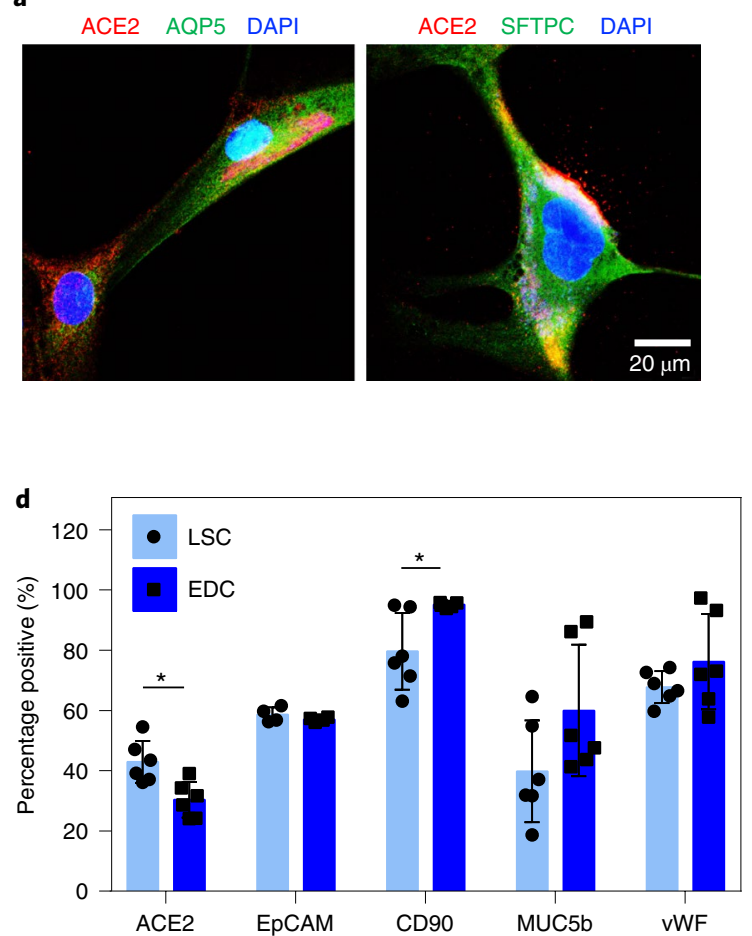

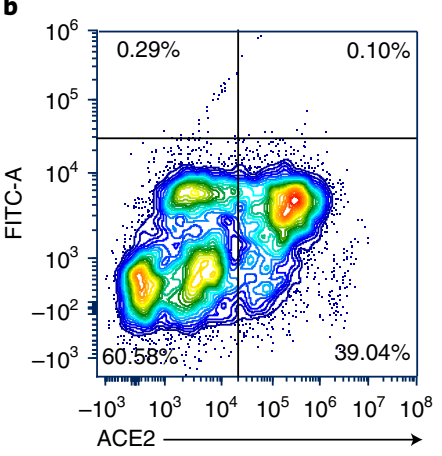

c

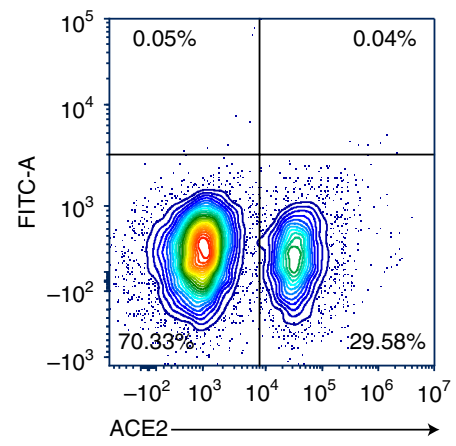

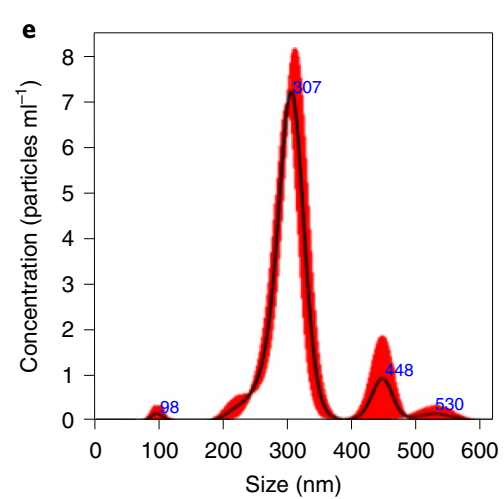

f

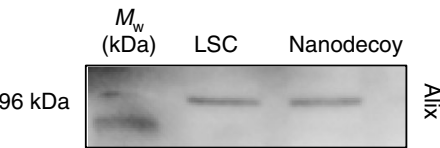

$100 \mathrm{kDa}$

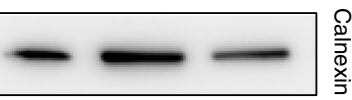

$43 \mathrm{kDa}$

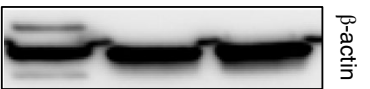

g

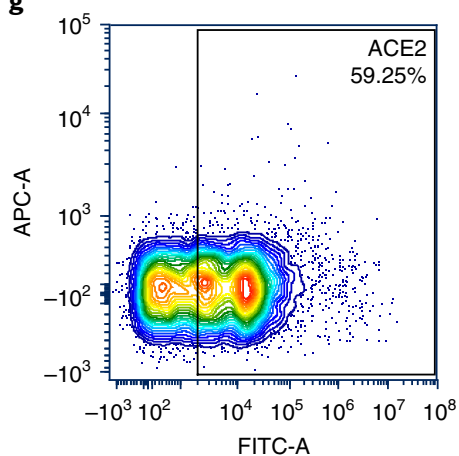

h

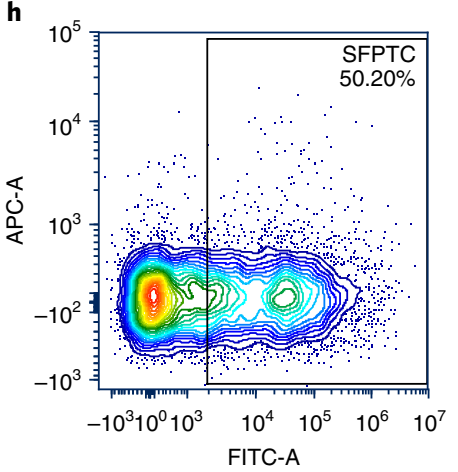

i Cell

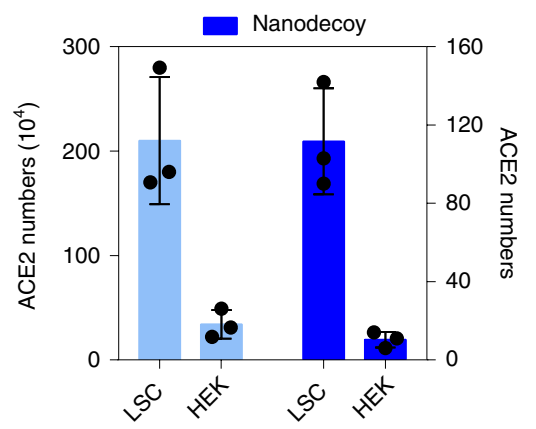

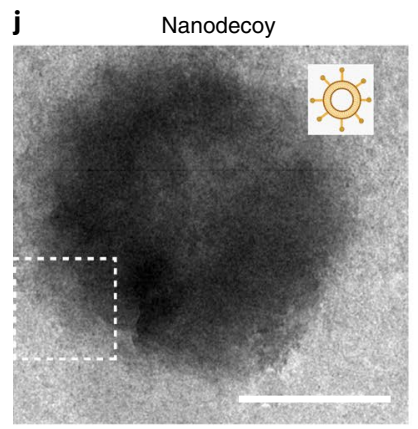
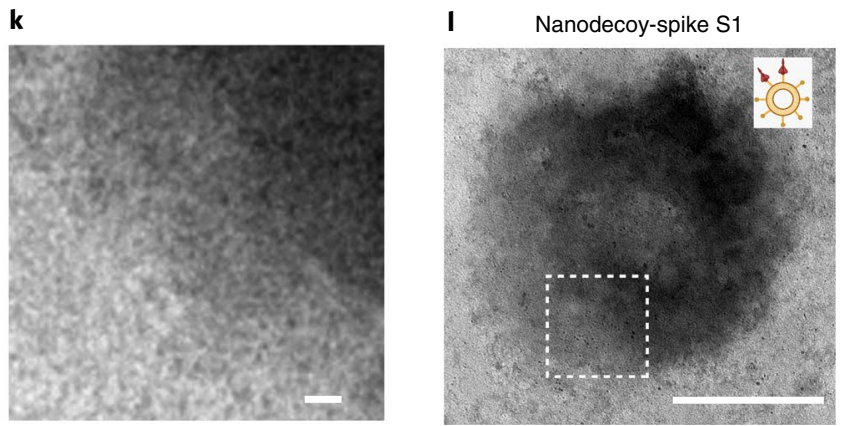

m

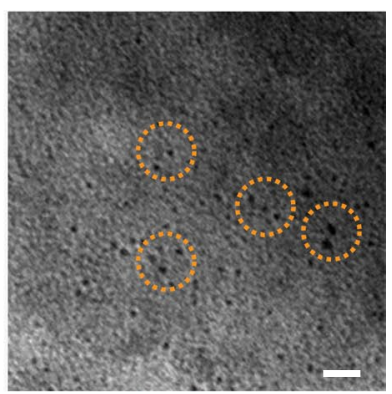

Fig. 1 | Characterizations of lung spheroid cell-derived nanodecoys. a, Representative confocal images of LSCs labelled with ACE2, AQP5 and SFTPC antibodies. DAPI (4',6-diamidino-2-phenylindole) was used to show nucleus. Three images were taken. b-d, Representative flow cytometry analysis of LSCs (b) and EDCs (c) for ACE2 expression and quantitative results of flow cytometry analysis of EDCs and LSCs for ACE2, EpCAM, CD90, MUC5b and von Willebrand factor (VWF) (d). FITC, fluorescein isothiocyanate. Data are shown as mean \pm SD, $n=4$ or 6 independent experiments. Statistical analysis was performed by two-way ANOVA with a Tukey post hoc test. See Supplementary Fig. 17 for gating strategies. e, Size measurement of nanodecoys using nanotracking particle analysis (Nanosight). Red shaded area, standard error. f, Western blot of Alix and Calnexin in LSC-nanodecoys and LSCs. g,h, Flow cytometry analysis showing the expressions of ACE2 ( $\mathbf{g}$ ) and the type II pneumocyte marker SFPTC (h) on LSC-nanodecoys. See Supplementary Fig. 17 for gating strategies. $\mathbf{i}$, Measurement of ACE2 numbers on both cells and nanodecoys. HEK indicates HEK293. Data are shown as mean \pm SD, $n=3$ independent experiments. j,k, TEM images showing naked nanodecoys (j) and enlarged figure ( $\mathbf{k})$. I,m, TEM images showing spike S1-bound nanodecoys (I) and enlarged figure ( $\mathbf{m})$. Spike S1 was detected using gold-nanoparticle-labelled secondary antibodies with diameters of $10 \mathrm{~nm}$. Scale bar in $\mathbf{j}$ and $\mathbf{i}, 200$ $\mathrm{nm}$; scale bar in $\mathbf{k}$ and $\mathbf{m}, 20 \mathrm{~nm}$. Cartoon pictures (insets in $\mathbf{j}$ and $\mathbf{I}$ ) were created with BioRender.com. 

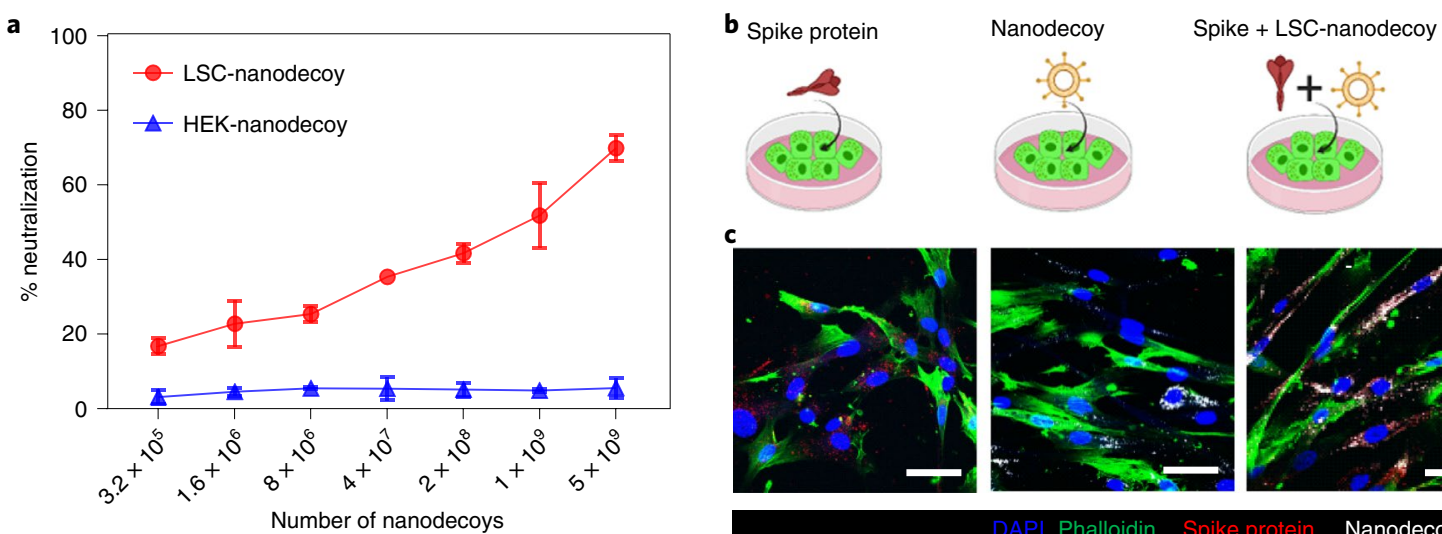

Spike + HEK-nanodecoy
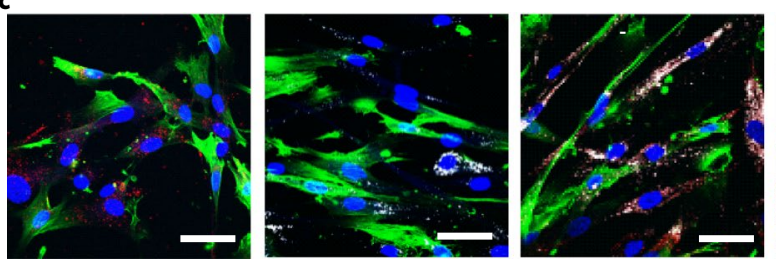

d

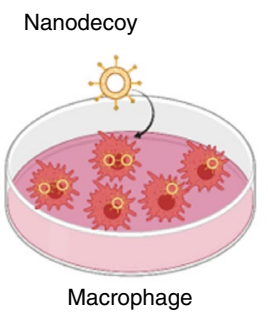

e

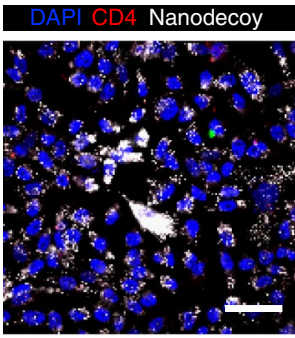

f

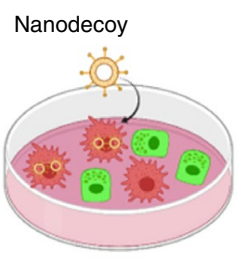

Macrophage+LSC g

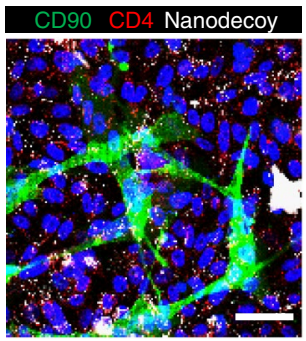

h

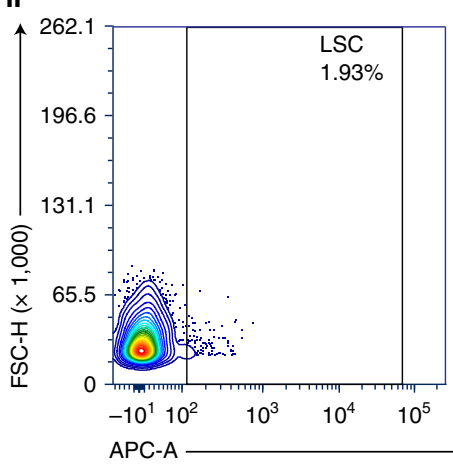

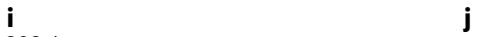

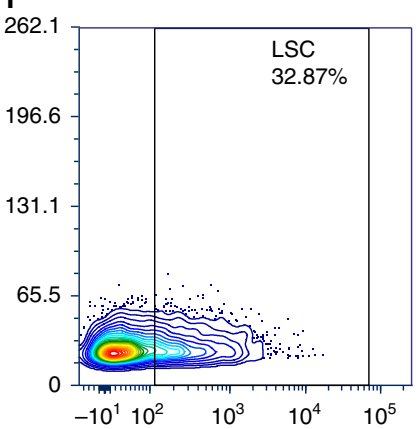

j

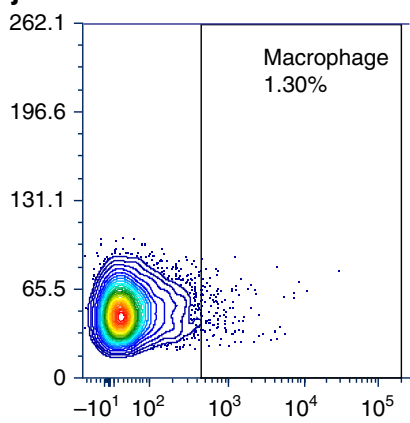

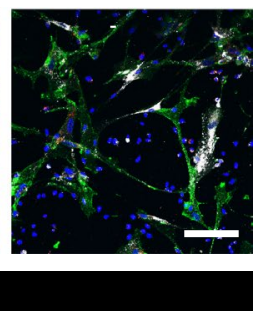
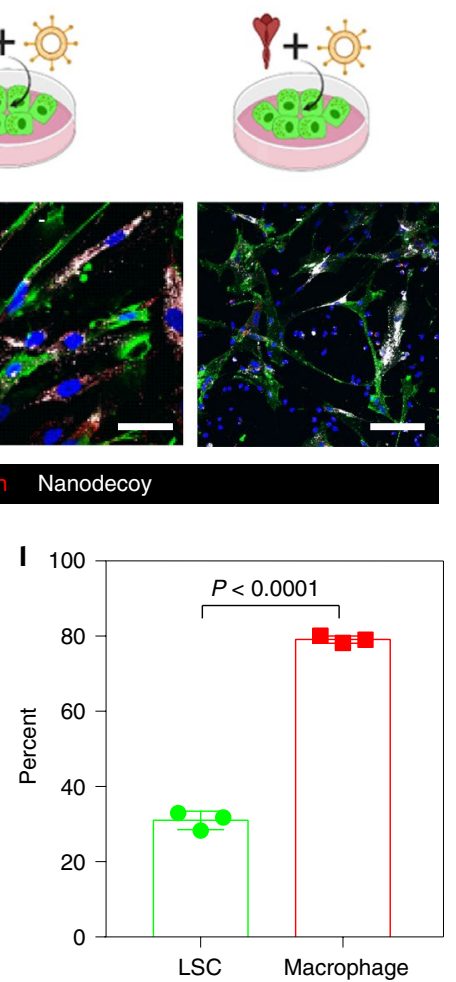

k

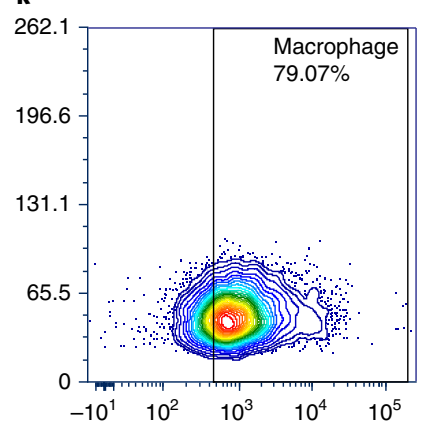

Fig. 2 | Neutralization of spike S1 by nanodecoys. a, Dose-dependent neutralization of spike S1 by LSC- or HEK-nanodecoys. Data are shown as mean \pm SD, $n=3$ independent experiments. b. Schematic illustrating the experimental design. c, Interaction of spike S1 (red) and nanodecoys (white) when co-cultured with lung cells (green). d, Schematic illustrating the experimental design. e, Representative confocal images showing internalization of nanodecoys by macrophages (CD4, red). Four images were taken. $\mathbf{f}, \mathbf{g}$, Schematic illustrating the co-culture experiment (f) and confocal images (g)

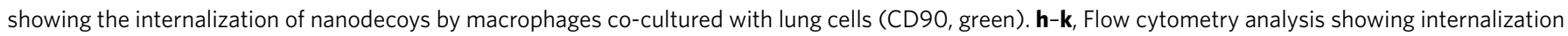
of DiD-labelled nanodecoys by LSCs (h,i) and macrophages (j,k) and the corresponding quantitation (I). FSC-H, forward scatter height. PBS was used as a control group for LSCs (i) and macrophages (j). See Supplementary Fig. 18a for gating strategies. Data are shown as mean \pm SD, $n=3$ independent

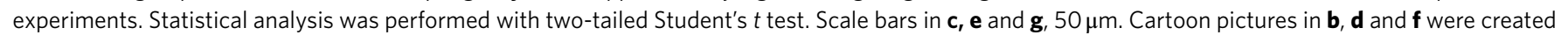
with BioRender.com.

products. Moreover, this nanodecoy technology is highly translatable because the parental cells are currently in the early clinical trial stage as a potential treatment for pulmonary fibrosis ${ }^{13}$.

\section{Fabrication of LSC-nanodecoys}

The overall rationale of our nanodecoy design is shown in Supplementary Fig. 1. First, LSCs and their parent cells, lung explant-derived cells (EDCs), were screened for ACE2 expression to determine the optimal cell types for nanodecoy fabrication. LSCs and EDCs were analysed by immunostaining (Fig. 1a and Supplementary Fig. 2), immunoblotting and flow cytometry (Fig. 1b,c and Supplementary Fig. 3) for ACE2 expression. In addition, the ACE2 expression levels of HEK293 and human lung fibroblasts were studied using immunoblotting and flow cytometry as controls (Supplementary Fig. 3). LSCs were found to have higher ACE2 expression levels than the other cell types, including their parent cell EDCs. In comparison, HEK293 and fibroblasts had visibly lower ACE2 expression. Consistent with previous studies, confocal imaging showed that ACE2 was present on the membrane of aquaporin 5-positive (AQP5 $5^{+}$type I pneumocytes and surfactant-associated protein C-positive $\left(\mathrm{SFTPC}^{+}\right)$-type II pneumocytes (Fig. 1a), two subpopulations of LSCs ${ }^{5,14}$. Analysis found that ACE2 was coexpressed with other LSC makers such as epithelial cell adhesion molecule (EpCAM), CD90 and mucin 
a

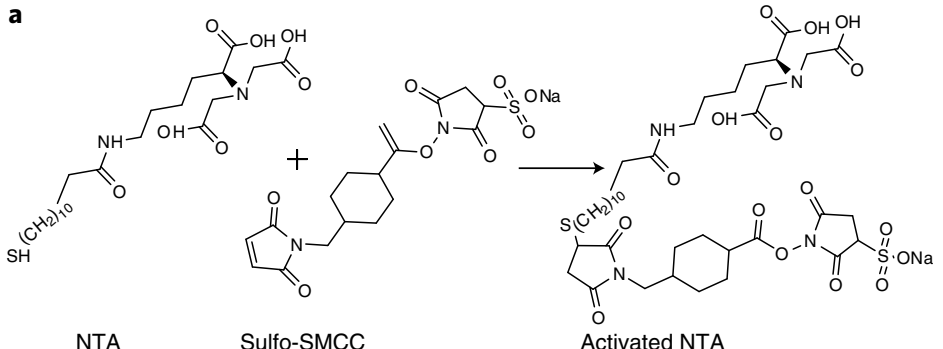

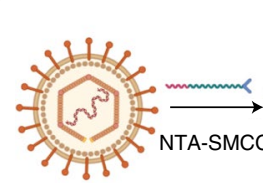

Lentivirus

c

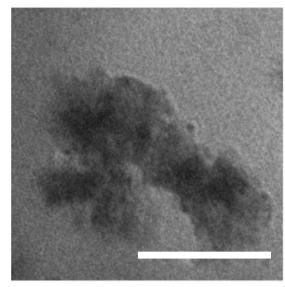

d

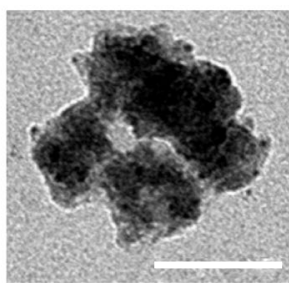

SARS-CoV-2 mimic

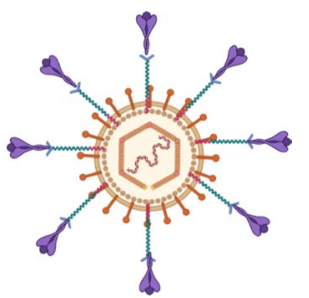

e

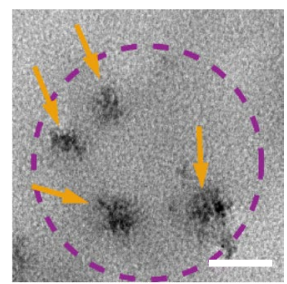

$\mathbf{f}$

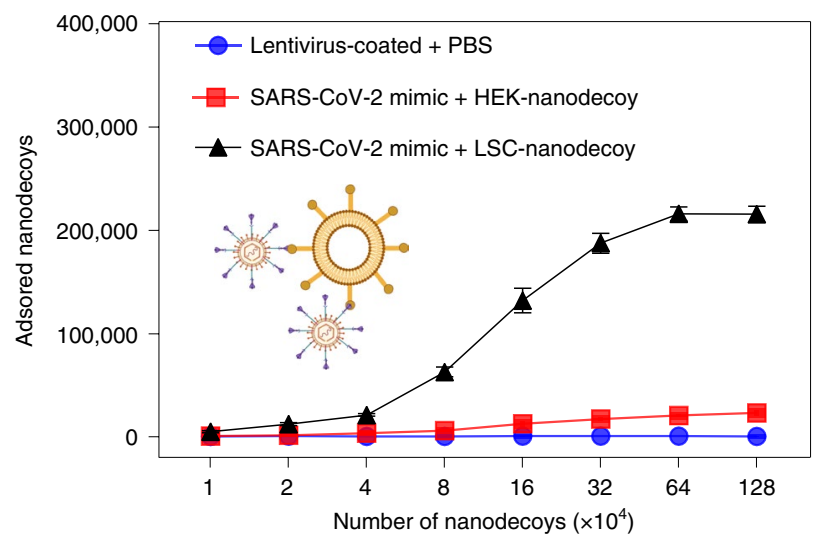

g

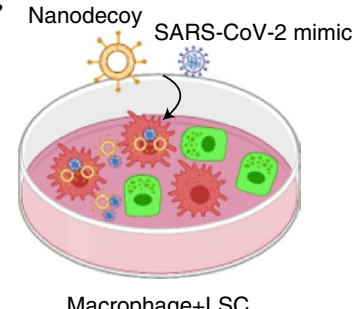

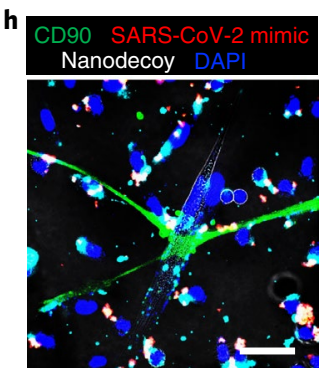
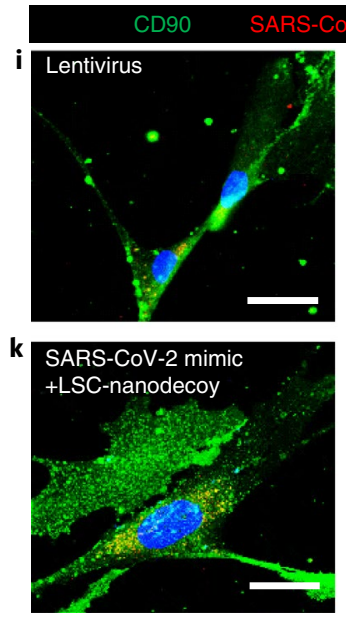

m

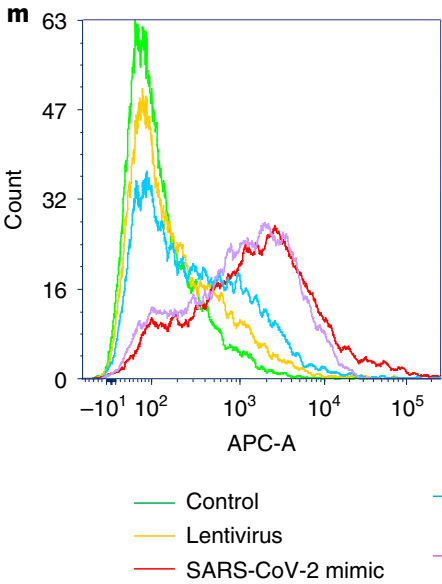

- SARS-CoV-2 mimic n

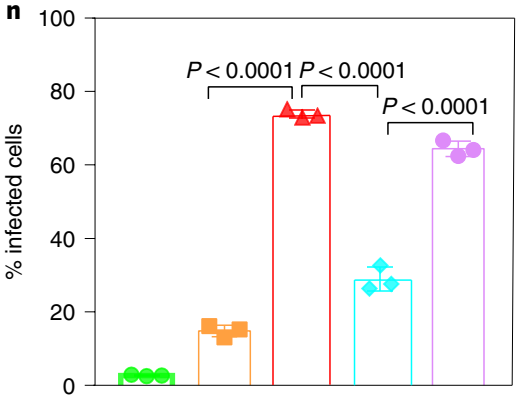

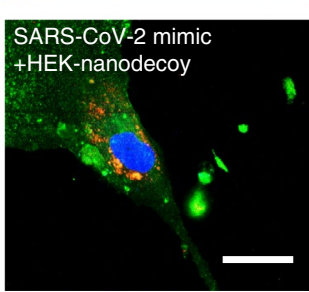

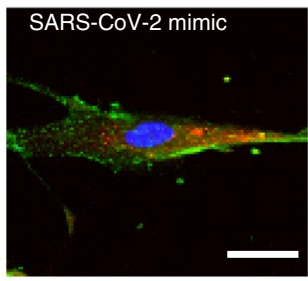

_ SARS-CoV-2 mimic + LSC-nanodecoy

- SARS-CoV-2 mimic + HEK-nanodecoy

Fig. 3 | Neutralization of SARS-CoV-2-mimicking viruses by nanodecoys. $\mathbf{a}$, Synthesis of activated NTA and its chemical structure. $\mathbf{b}$, Schematic illustrating the modification of lentivirus with spike S1 to generate a SARS-CoV-2 mimic. c-e, TEM images showing lentivirus (c), SARS-CoV-2 mimic (d) and spike S1 on lentivirus using gold-nanoparticle-labelled secondary antibodies with diameters of $10 \mathrm{~nm}$, in which SARS-CoV-2-mimicking viruses (yellow arrows) were attached to a nanodecoy (dotted circle) (e). Scale bar, $100 \mathrm{~nm}$. f, Neutralization assay of SARS-CoV-2 mimics by nanodecoys. Data are shown as mean \pm SD, $n=4$ independent experiments. $\mathbf{g}$, Schematic showing the experimental design. $\mathbf{h}$, Nanodecoys (white) neutralize SARS-CoV-2 mimics (red) in a co-culture with lung cells (green) and macrophages. Scale bar, $50 \mu \mathrm{m}$. i-l, Representative confocal images of lentivirus (i), SARS-CoV-2 mimic (j), SARS-CoV-2 mimic plus LSC-nanodecoy (k) and SARS-CoV-2 mimic plus HEK-nanodecoy (I) entry into the lung cells (green). At least three images were taken. Scale bar, $50 \mu \mathrm{m} . \mathbf{m}, \mathbf{n}$. Flow cytometry analysis showing that nanodecoys inhibit the entry of SARS-CoV-2 mimic virus into the lung cells ( $\mathbf{m}$ ), and the corresponding quantitation (n). See Supplementary Fig. 18b for gating strategies. Data are shown as mean $\pm S D, n=3$ independent experiments. Statistical analysis was performed by one-way ANOVA with a Tukey post hoc test. Cartoon pictures in $\mathbf{b}, \mathbf{f}$ and $\mathbf{g}$ were created with BioRender.com.

5b (MUC5b) (Fig. 1d and Supplementary Figs. 4 and 5). Previous studies have indicated that $83 \%$ of ACE2-expressing cells in lung tissue are type II pneumocytes, suggesting that the lungs are the most vulnerable target organs of the SARS-CoV-2 virus ${ }^{15}$. Thus, our results demonstrated that, as primary resident lung cells, LSCs might serve as an ideal cell type to generate nanodecoys 
a

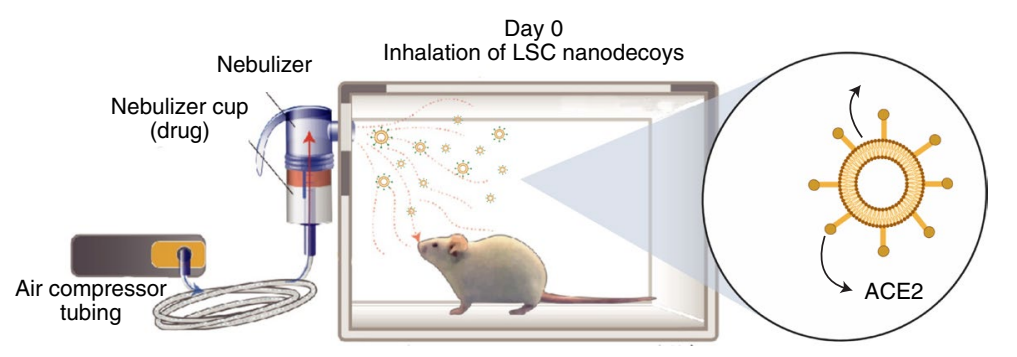

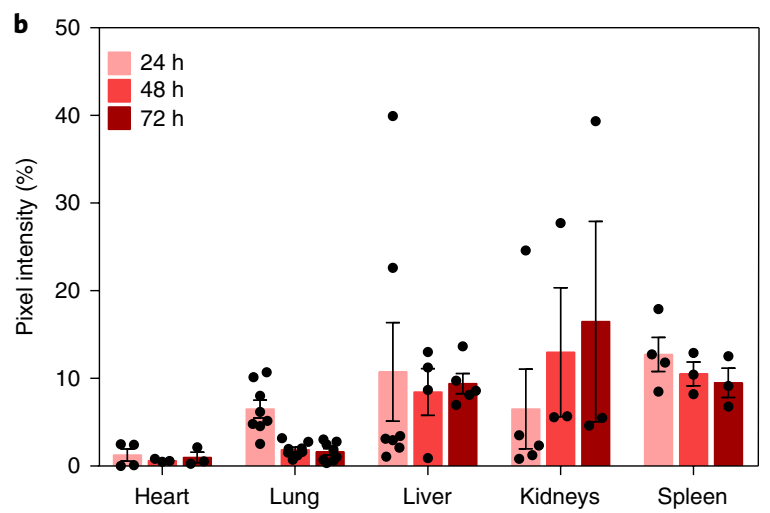
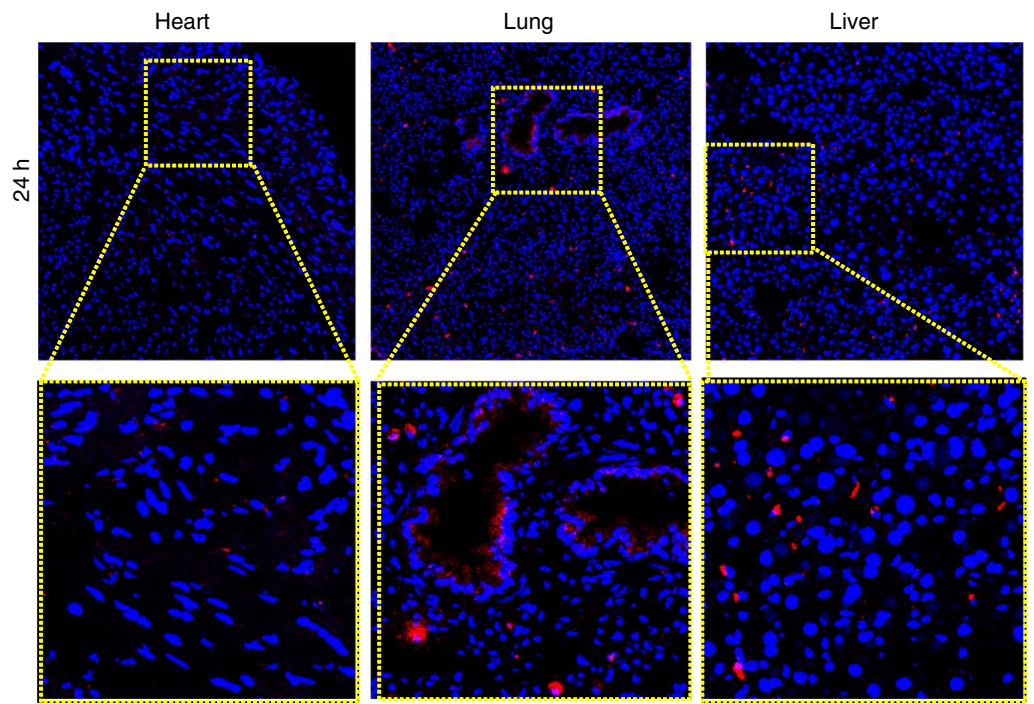

d

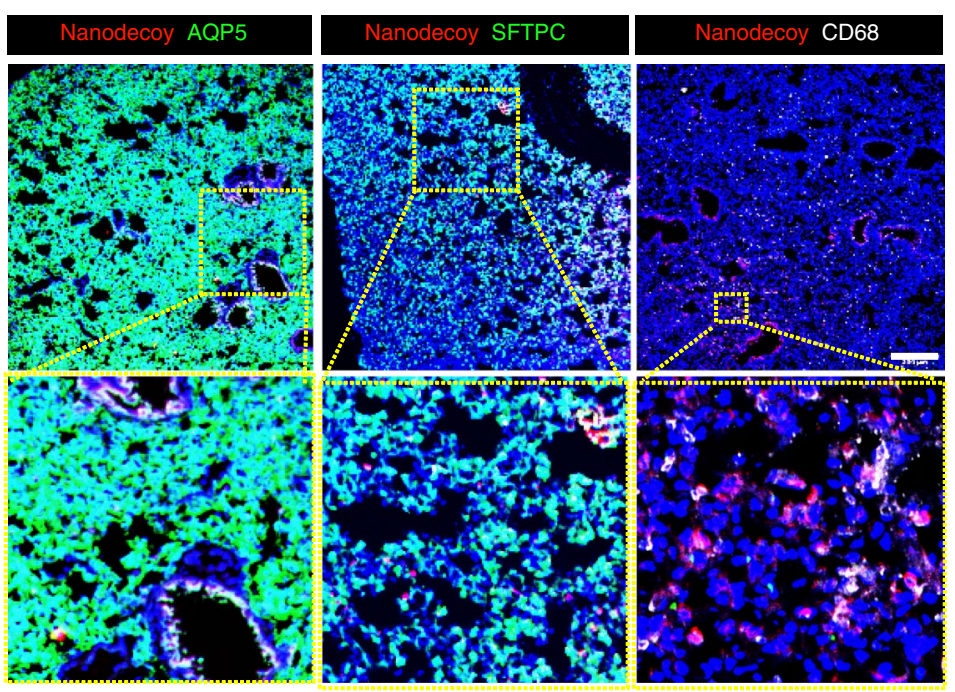

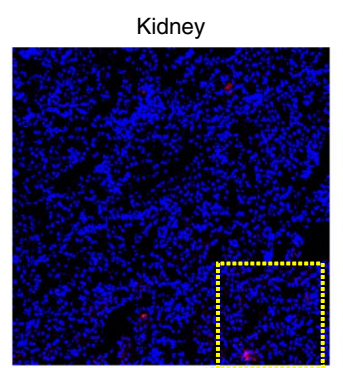
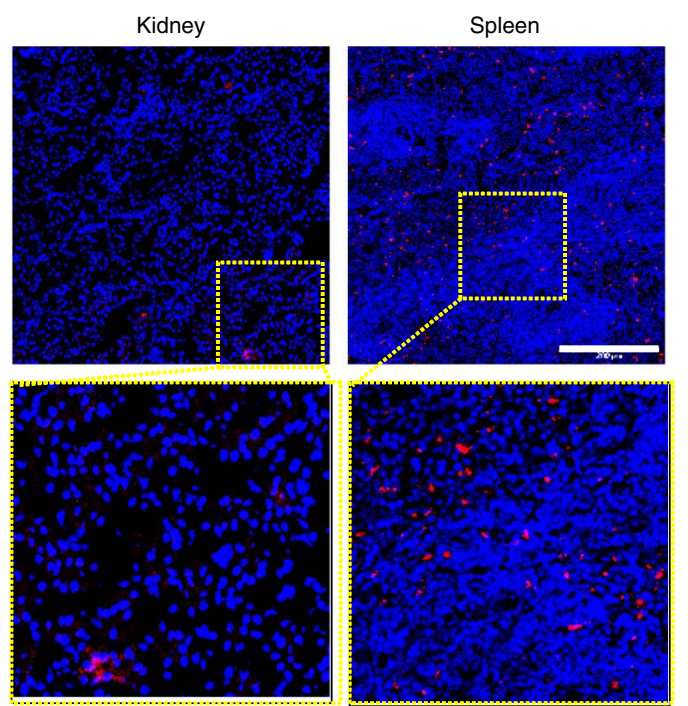

e

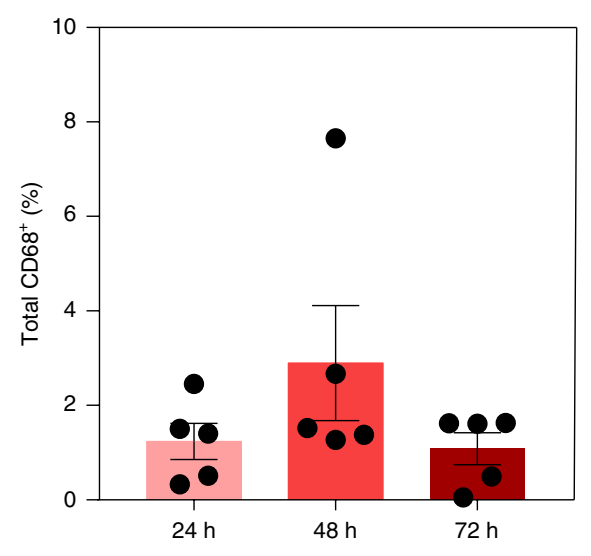

Fig. 4 | Biodistribution of nanodecoys after inhalation. a, Schematic showing study design of nanodecoy inhalation in CD1 mice. Created with BioRender. com. b. Corresponding quantitative results from $\mathbf{c}$ of DiD-labelled nanodecoys in heart, lung, liver, kidney and spleen tissues. Data are shown as mean \pm SD, $n=3$ animals. At least three images were taken. c, Representative confocal images of DiD-labelled nanodecoys (red) in tissue sections. d, Representative confocal images showing nanodecoys in lung tissues co-localizing with lung cells (AQP5, SFTPC) and macrophages (CD68) $24 \mathrm{~h}$ post-inhalation. Five images were taken. e, Quantification of the percentage of nanodecoy-positive macrophages (CD68). Data are shown as mean \pm SD, $n=3$ animals. Scale bar in $\mathbf{c}$ and $\mathbf{d}, 200 \mu \mathrm{m}$.

with high levels of ACE2 expression. In contrast, HEK293 cells were used as a control for preparing nanodecoys with a low level of ACE2 expression.
LSC and HEK293 membrane nanovesicles (nanodecoys) were generated by serial extrusion of LSCs or HEK293 cells through polycarbonate membranes with pore sizes of $5 \mu \mathrm{m}, 1 \mu \mathrm{m}$ and finally 
a

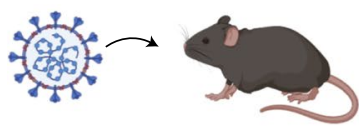

Inhalation of SARS-CoV-2 mimics

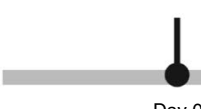

Day 0

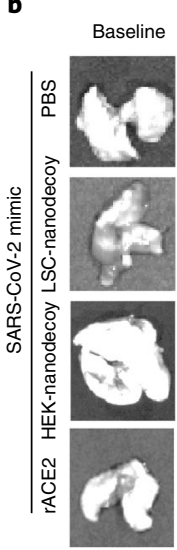

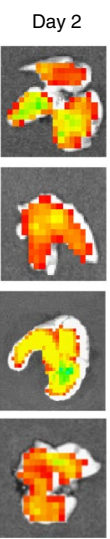

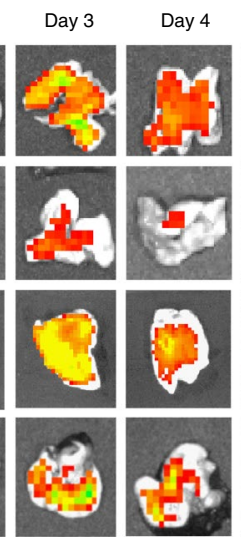

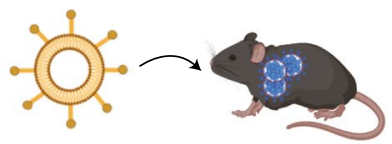

Inhalation of nanodecoys

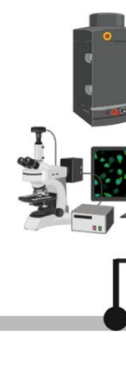

IVIS/ex vivo imaging

Harvest organs for IHC

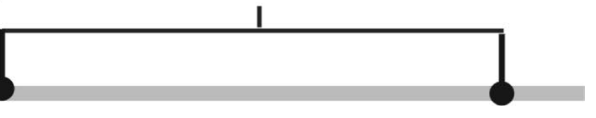

Days 2-6

C

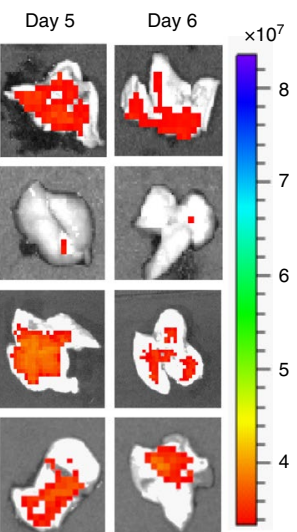

C SARS-CoV-2 mimic + PBS SARS-CoV-2 mimic + HEK-nanodecoy SARS-CoV-2 mimic + rACE2 SARS-CoV-2 mimic + LSC-nanodecoy

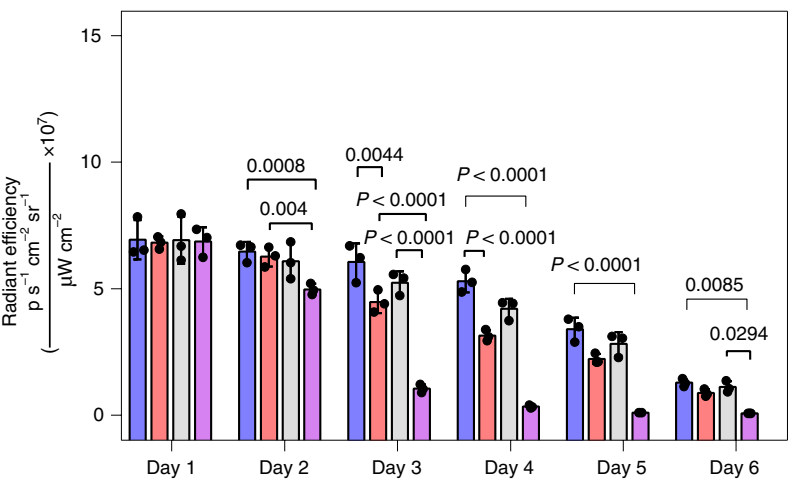

d
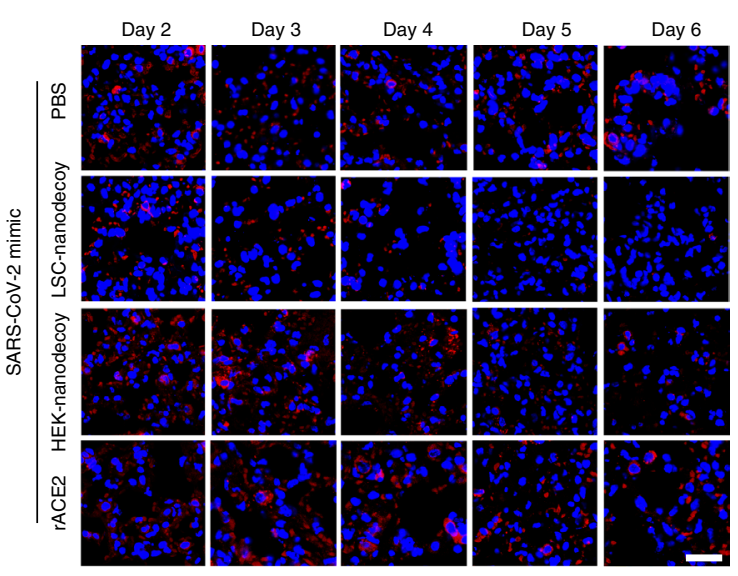

f

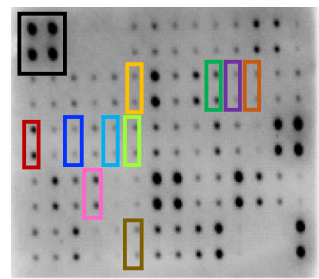

g

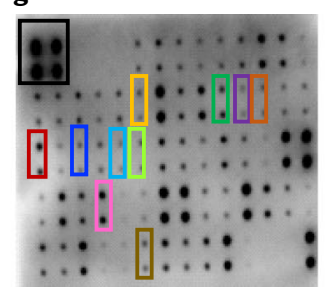

h

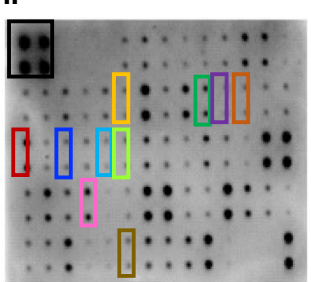

e

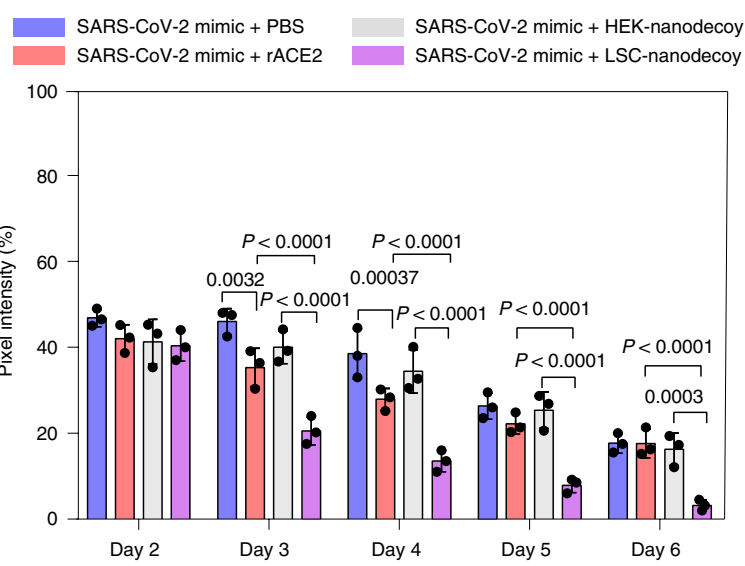

Fig. 5 | Nanodecoy inhalation accelerates the clearance of the SARS-CoV-2 mimic viruses in a mouse model. a, Schematic showing the animal study design. Created with BioRender.com. b. Representative ex vivo IVIS imaging of lung tissues from mice with various treatments. $n=3$ animals per group. Colour scale shows radiant efficiency (same units as c). c, Quantification of fluorescence intensities of SARS-CoV-2 mimics from the imaging data in b. Data are shown as mean $\pm S D, n=3$ animals per group. Statistical analysis was performed by two-way ANOVA with a Tukey post hoc test for multiple comparisons. p, photons. d, Representative confocal images of AF647-labelled SARS-CoV-2 mimics (red) in lung sections. Scale bar, $50 \mu \mathrm{m}$. $n=3$ animals per group. Three images were taken for each animal. e, Corresponding semiquantitative analysis of AF647-labelled SARS-CoV- 2 mimics in lung tissues. Data are shown as mean \pm SD, $n=3$ animals per group. Statistical analysis was performed by two-way ANOVA with a Tukey post hoc test for multiple comparisons. f-j, Cytokine array analysis of various inflammatory cytokines (j) in the serum $3 \mathrm{~d}$ after treatment: control (f), LSC-nanodecoy (g), SARS-CoV-2 mimic (h) and SARS-CoV-2 mimic plus LSC-decoy (i). IFN- $\gamma$, interferon $\gamma$; IL, interleukin; M-CSF, macrophage colony-stimulating factor; POS, positive control; TNF- $\alpha$, tumor necrosis factor $\alpha$.

$0.4 \mu \mathrm{m}$ with a commercial extruder. The obtained LSC-nanodecoys were characterized by nanoparticle tracking analysis, which showed a homogeneous nanoparticle population with an average size of
$320 \mathrm{~nm}$ and an average concentration of $5.51 \times 10^{10}$ particles $\mathrm{ml}^{-1}$ produced from $5 \times 10^{6}$ cells (Fig. 1e). In other words, on average, one LSC generated 11,020 nanodecoys. Because whole cells were 
a

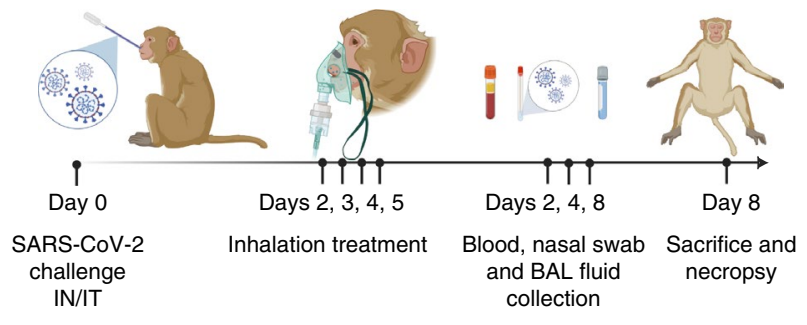

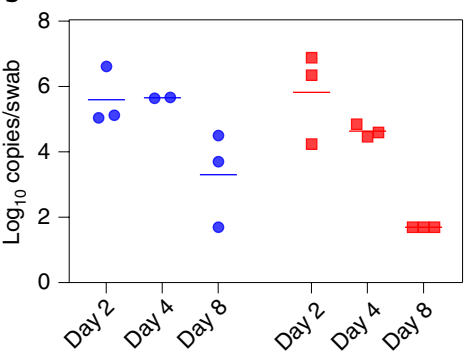

c

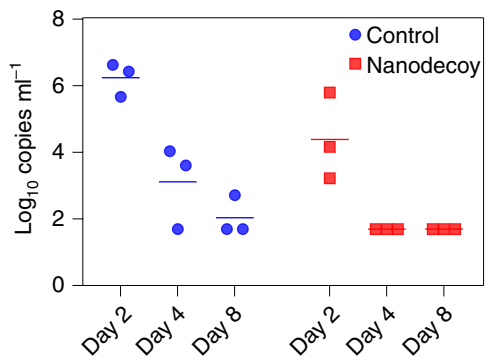

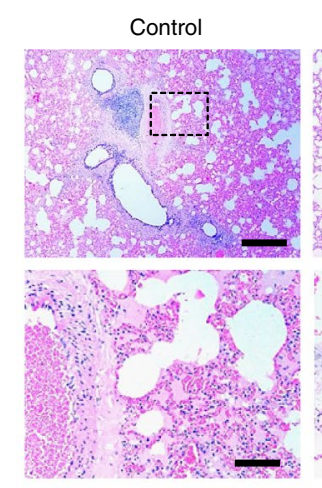

Nanodecoy

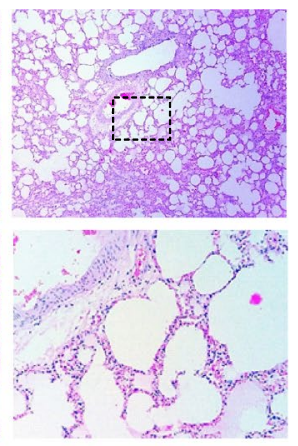

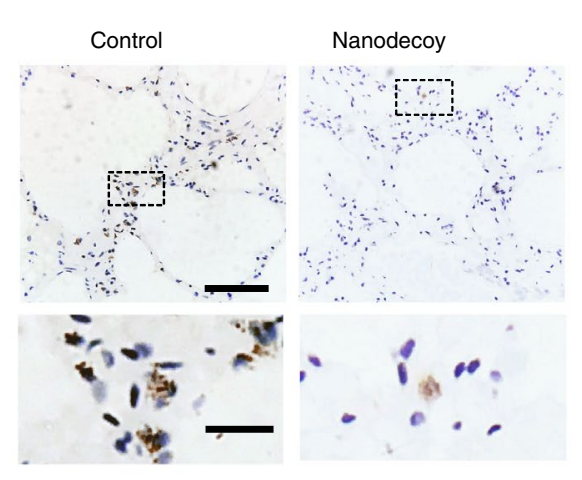
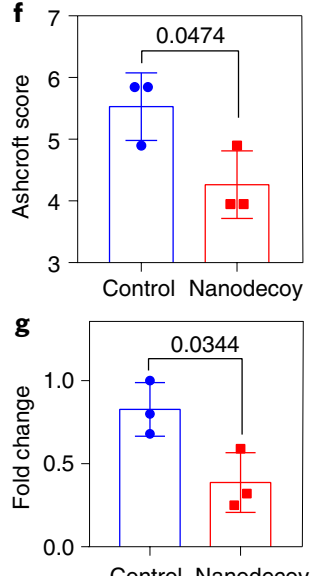
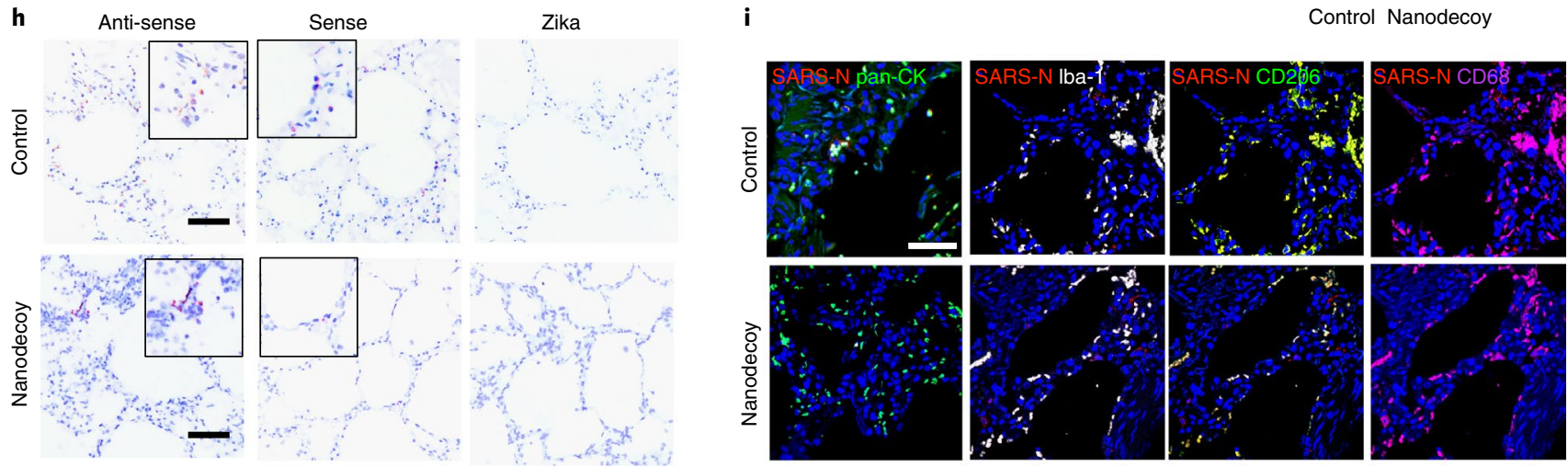

Fig. 6 | LSC-nanodecoy inhalation treats SARS-CoV-2 infection in cynomolgus macaques. a, Schematic depicting the cynomolgus macaque study design. IN, intranasal. IT, intratracheal. Created with BioRender.com. b,c, Viral sgRNA copies/swab in NSs (b) and BAL (c) at various time points following challenge. Each dot represents data from one animal. $n=3$ animals per group. d, Representative H\&E images of fixed lung tissues from SARS-CoV2 -infected cynomolgus macaques. At least three images were taken per animal. Scale bars, $500 \mu \mathrm{m}$ (top), $100 \mu \mathrm{m}$ (bottom). e, Representative images of SARS-N IHC staining in fixed lung tissues from SARS-CoV-2-infected cynomolgus macaques treated with control or LSC-nanodecoys $8 \mathrm{~d}$ post-viral challenging. $n=3$ animals per group. Three images were taken for each animal. Scale bars, $100 \mu \mathrm{m}$ (top), $20 \mu \mathrm{m}$ (bottom). f, Quantification of lung fibrosis of infected cynomolgus macaques by Ashcroft scoring; each dot represents data from one animal. Data are shown as mean \pm SD, $n=3$ animals per group. Statistical analysis was performed by two-tailed Student's $t$ test. Ashcroft scoring was performed blindly. $\mathbf{g}$, Quantitation of positive SARS-N numbers in lung tissues of infected cynomolgus macaques. Each dot represents data from one animal. Data are shown as mean $\pm S D, n=3$ animals per group. Statistical analysis was performed by two-tailed Student's $t$ test. $\mathbf{h}$, Representative images of RNAscope in situ hybridization detection of vRNA in infected cynomolgus macaques. Zika served as a control probe. $n=3$ animals per group. Three images were taken for each animal. Scale bar, $100 \mu \mathrm{m}$. $\mathbf{i}$, Representative immunofluorescence images of SARS-N (red), pan-CK (green), Iba-1 (greyscale), CD68 (green), CD206 (magenta) and DAPI (blue). Scale bar, $50 \mu \mathrm{m} . n=3$ animals per group. Three images were taken for each animal.

used to prepare the nanodecoys, we suspected that the nanodecoys were not exclusively generated from the plasma membranes but also from intracellular membranes. To confirm our hypothesis, we also studied the intracellular components of the nanodecoys by testing for Alix (a phylogenetically conserved cytosolic scaffold protein) and Calnexin (a marker of endoplasmic reticulum). Results showed that these two intracellular markers were detected, which supported our claims (Fig. 1f). Flow cytometry analysis confirmed the preservation of ACE2 on the surface of the nanodecoys (Fig. 1g) as well as the type II pneumocyte marker SFTPC (Fig. 1h). Moreover, we studied the quantity of ACE2 on both LSCs and HEK293 cells and their nanodecoys by enzyme-linked immunosorbent assay (ELISA). The abundance of ACE2 was determined to be $2.1 \times 10^{6}$ receptors per LSC and 112 receptors per LSC-nanodecoy. In stark contrast, only $3.4 \times 10^{5}$ and 10 ACE2 receptors were found to be present on each HEK293 cell and HEK-nanodecoy, respectively (Fig. 1i). Furthermore, transmission electron microscope (TEM) images revealed the spherical morphology of nanodecoys (Fig. 1j,k). 


\section{Nanodecoys bind and neutralize spike S1 in vitro}

Having demonstrated the presence of ACE2 on the nanodecoys, we then tested their binding ability to the SARS-CoV-2 spike protein. Spike $S 1$ of the spike protein contains a receptor-binding domain that specifically recognizes ACE2. Therefore, we first confirmed that spike S1 could bind to the nanodecoys by TEM with immunogold labelling (Fig. 11,m). In a dose-responsive manner, $50 \%$ of spike S1 $\left(6.5 \times 10^{10}\right)$ was captured and bound by $10^{9}$ LSC-nanodecoys, whereas nanodecoys derived from HEK293 cells failed to bind spike S1 (Fig. 2a). We then examined the binding potency of LSC- and HEK293-nanodecoys using lung cell-based assays (Fig. 2b). Spike S1 was found to bind to lung cells after $4 \mathrm{~h}$ of incubation (Fig. 2c). 1,1'-Dioctadecyl3,3,3',3'-tetramethylindodicarbocyanine,4-chlorobenzenesulfonate salt (DID)-labelled LSC-nanodecoys co-localized with spike S1, while HEK293-nanodecoys did not, suggesting that the LSC-nanodecoys could recognize and competitively bind spike S1. Additionally, macrophages had a greater internalization efficiency of the nanodecoys than the lung cells did (Fig. $2 \mathrm{~d}-\mathrm{g}$ ), indicating the potential clearance of nanodecoys and their neutralized SARS-CoV-2 by macrophages and/or other immune cells, which was confirmed by flow cytometry analysis (Fig. 2h-1). Furthermore, both peripheral blood and alveolar macrophages had a similar internalization rate of LSC-nanodecoys (Supplementary Fig. 6).

\section{Nanodecoys bind and neutralize SARS-CoV-2 mimics}

Next, we fabricated a spike S1 virus to mimic SARS-CoV-2 by modifying a lentivirus to have spike $\mathrm{S} 1$ on its surface. Lentiviruses were first modified with Ni nitrilotriacetate (Ni-NTA) (Fig. 3a), and His-tagged spike S1 was then conjugated onto the lentivirus through the interaction of $\mathrm{Ni}$ with the His tag to generate this SARS-CoV-2 mimic (Fig. 3b). Immunogold labelling was used to confirm spike $\mathrm{S} 1$ on the SARS-CoV-2 mimics. TEM imaging visualized the bare lentivirus (Fig. 3c), SARS-CoV-2 mimic (Fig. 3d) and the nanodecoy-bound SARS-CoV-2 mimics, shown by the presence of spike $\mathrm{S} 1$ on the surface of the modified lentivirus together with the nanodecoy (Fig. 3e), indicating the SARS-CoV-2 mimics were fabricated successfully. Our examination indicated that there were approximately 6,900 spike S1s per SARS-CoV-2 mimic virus. We found that $2.16 \times 10^{5}$ LSC-nanodecoys could bind $5 \times 10^{5}$ SARS-CoV-2 mimics (2.31 SARS-CoV-2 mimics per nanodecoy) while HEK293-nanodecoys showed a lower binding efficiency to SARS-CoV-2 mimics, which was possibly due to the low ACE2 level (Fig. 3f). This binding interaction is specific because the control lentivirus (without spike S1) had low affinity to LSC-nanodecoys. We co-cultured macrophages and LSCs (Fig. 3g) and found SARS-CoV-2 mimics were recognized by LSC-nanodecoys and internalized by macrophages after $4 \mathrm{~h}$ in co-culture (Fig. $3 \mathrm{~h}$ ). We then studied the intracellular distribution of the mimics; confocal imaging showed some of the mimics within the lysosomes while others resided in the cytoplasm (Supplementary Fig. 7). In addition, lentiviruses before and after modification had a negligible difference in internalization by LSCs (Supplementary Fig. 8). We next studied the antiviral protection effects of nanodecoys on LSCs. Immunocytochemistry (Fig. 3i-1) and flow cytometry (Fig. $3 \mathrm{~m}, \mathrm{n}$ ) confirmed that LSC-nanodecoys could block the entry of SARS-CoV-2 mimics in host cells, but HEK293-nanodecoys could not. Naive lentiviruses were not efficient in entering lung cells (14.8\% infection rate) (Fig. 3i). However, spike S1-modified lentiviruses (SARS-CoV-2 mimics) promoted entry into host cells efficiently (73.8\% infection rate) (Fig. 3j), whereas compared with HEK-nanodecoys, LSC-nanodecoys significantly decreased the internalization of SARS-CoV-2 mimics (from $73.8 \%$ to $28.8 \%$ ) (Fig. $3 \mathrm{k}, 1)$. In addition, we studied the dose-dependent blocking effect by LSC-nanodecoys. Flow cytometry analysis showed that increasing doses of LSC-nanodecoys blocked more virus entry into lung cells in a dose-dependent manner (Supplementary Fig. 9). Together these results suggest LSC-nanodecoys could protect the host cells from infection by SARS-CoV-2 mimics.

We next studied the biodistribution of LSC-nanodecoys in mice after inhalation. DiD-labelled nanodecoys were administered to mice by inhalation using a commercially available portable nebulizer for clinical relevance at a dose of $1 \times 10^{10}$ nanodecoys per $\mathrm{kg}$ of body weight (Fig. 4a). As shown in Fig. 4b,c and Supplementary Fig. 10 , nanodecoys could still be found in the lungs $72 \mathrm{~h}$ after a single inhalation treatment. In addition to the lungs, the nanodecoys were also detected in the liver, kidney and spleen, indicating clearance via the reticuloendothelial system as well as the metabolization of the nanodecoys through the body. Moreover, inhalation of nanodecoys had no significant effect on $\mathrm{CD}^{+} 8^{+}$macrophage infiltration, indicating biocompatibility (Supplementary Fig. 11). Although some nanodecoys co-localized with $\mathrm{APQ}^{+}$(type I) and SFTPC ${ }^{+}$ (type II) cells (Fig. 4d and Supplementary Fig. 12), the majority of nanodecoys were co-localized in macrophages (Fig. 4e) $24 \mathrm{~h}$ after inhalation.

We tested whether inhaled LSC-nanodecoys could accelerate the clearance of SARS-CoV-2 mimics in a mouse model (Fig. 5a). To mimic infection in human patients, we allowed the mice to receive the SARS-CoV-2 mimics before initiating administration of the therapeutic nanodecoys. Because treatment started $24 \mathrm{~h}$ after viral exposure, not all of the SARS-CoV-2 mimics were intracellular; therefore, nanodecoys could block the viral mimics from entering the cells further. As for the intracellular SARS-CoV-2 mimics, the nanodecoys that were internalized by cells could capture them, avoiding further infection. Ex vivo imaging (Fig. 5b,c) indicated that the amounts of SARS-CoV-2 mimics were significantly reduced following inhalation of LSC-nanodecoys. Inhalation of the free form of recombinant ACE2 (rACE2)- or HEK293-nanodecoys was found to be ineffective. Confocal microscopy confirmed that inhalation of LSC-nanodecoys accelerated the clearance of SARS-CoV-2 mimics (Fig. 5d,e). Cytokine array analysis (Fig. 5f-j) suggested that nanodecoy inhalation did not elevate proinflammatory cytokines as compared to the control group. Furthermore, haematoxylin \& eosin $(\mathrm{H} \& \mathrm{E})$ staining of all major organs, haematology and biochemical parameters indicated no apparent abnormality or adverse effects with LSC- or HEK293-nanodecoy inhalation (Supplementary Figs. 13 and 14).

\section{Nanodecoy therapy in a primate model of COVID-19}

We then performed a pilot non-human primate study to evaluate the safety and preliminary therapeutic efficacy of LSC-nanodecoys. The macaque model can recapitulate many clinical symptoms of SARS-CoV-2 infection and shows a robust viral replication in the upper and lower respiratory tracts. Six cynomolgus macaques (Macaca fascicularis) were challenged with SARS-CoV-2 by intranasal and intratracheal routes (Fig. 6a). Following challenge, the animals were randomized into two treatment arms: inhalation of either PBS or LSC-nanodecoys (at a dose of $10^{10}$ particles per $\mathrm{kg}$ of body weight) at days 2, 3, 4 and 5 post-challenge. Viral loads in bronchoalveolar lavage (BAL) and nasal swabs (NSs) were assessed by real-time PCR with reverse transcription specific for viral subgenomic RNA (sgRNA, indicative of virus replication). As a result, high levels of sgRNA were observed in the control animals with a median peak of $6.243 \log _{10}$ RNA copies $\mathrm{ml}^{-1}$ in BAL and a median peak of $5.595 \log _{10}$ RNA copies per swab in NSs on day 2 (Fig. 6b,c). sgRNA levels dramatically decreased in nanodecoy-treated animals, with $<1.70 \log _{10}$ reductions of median peak sgRNA in both BAL and NSs on day 8 following the challenge. Although sgRNA levels declined in both control and LSC-nanodecoy groups over time, LSC-nanodecoy treatment induced more rapid virus clearance. Negligible difference was observed between the two groups' haematology parameters (Supplementary Fig. 15). Interestingly, the 
temperature and body weight fluctuations in the LSC-nanodecoy group were not as drastic as those in control-treated animals (Supplementary Fig. 16).

At the end of the study, lung tissues of infected cynomolgus macaques were collected and evaluated by histopathology. On day 8 following challenge, multifocal regions of inflammation and evidence of viral pneumonia-including expansion of alveolar septae with mononuclear cell infiltrates, consolidation and oedema-were observed (Fig. 6d). Notably, LSC-nanodecoy treatment significantly reduced the numbers of polymorphonuclear cells and neutrophils as compared with the control group. In addition, Ashcroft score analysis revealed that LSC-nanodecoy treatment significantly decreased lung fibrosis (Fig. 6f). To detect and visualize the virus in lung tissues, SARS nucleocapsid protein (SARS-N) expression was evaluated by immunohistochemistry (IHC) staining. As shown in Fig. 6e,g, multifocal positive pneumocytes and alveolar septa were present in control-treated animals. In contrast, the levels of SARS-N protein were decreased substantially with the LSC-nanodecoy treatment. In addition, SARS-CoV-2 viral RNA (vRNA) was evaluated by in situ RNA hybridization (RNAscope). Compared with the control group, the levels of both positive-sense and negative-sense vRNA were diminished after LSC-nanodecoy treatment (Fig. 6h), indicative of the reduction of viral replication. The distribution of SARS-CoV-2 in lung tissue was assessed by co-staining SARS-N and pan-cytokeratin (pan-CK, to identify epithelial cells). We found that virus-infected cells greatly overlapped with pan-CK-positive cells (Fig. 6i), suggesting that they were alveolar epithelial cells. Additionally, foci of virus-infected cells were frequently associated with activated Iba- $1^{+}$ (ionized calcium binding adaptor as a pan-macrophage marker), $\mathrm{CD}^{+} 8^{+}$(monocyte/macrophage marker) and CD206 ${ }^{+}$(macrophage marker) macrophages (Fig. 6i). Consistent with IHC and RNAscope analysis, immunofluorescence results indicated that nanodecoys could decrease virus levels in lung tissues.

Previous studies have indicated that ACE2 is the host receptor for the novel coronavirus SARS-CoV-2 and that viral entry of SARS-CoV-2 depends on the binding of the viral spike S1 to ACE2 on the host cells ${ }^{16}$. Therefore, inhibiting the binding of spike S1 to ACE2 is a promising treatment strategy to combat COVID-19. Inspired by this discovery, some previous works have focused on blocking SARS-CoV-2 entry by using rACE2 protein, either alone or fused with an $\mathrm{Fc}$ fragment $(\mathrm{rACE} 2-\mathrm{Fc})^{17,18}$. However, those protein-based neutralization strategies are limited by their overall short half-life after administration. Furthermore, undesired dosage and distribution of extracellular ACE2 could cause unknown toxicity effects on the body ${ }^{17}$. In addition, except for ACE2, other components on cell membranes also play roles in virus docking; therefore, targeting ACE2 alone may not be the best strategy.

Previous works have shown several antimicrobial applications by utilizing cell-membrane-based nanodecoys ${ }^{19-21}$. For example, nanodecoys from Aedes albopictus (C6/36) cell-membrane-coated gelatin nanoparticles have been developed to trap Zika virus to prevent viral infection ${ }^{22}$. Also, T-cell-membrane-coated nanoparticles have been used as decoys for HIV neutralization because of the presence of T-cell surface antigens for HIV binding ${ }^{23}$. In addition to cellular-membrane-based nanodecoys, engineered liposomes have also been fabricated as decoy targets to sequester bacterial toxins produced during active infection in vivo ${ }^{24}$. Our strategy represents a potential nanodecoy treatment for COVID-19 (Supplementary Fig. 1). The nanodecoys could be generated from human lung cells on a large scale using commercially available extrusion devices. They not only express natural human ACE2 but also represent a mimic of human lung cells, which are the main targets of SARS-CoV-2.

Two concerns of drug development are potential off-target effects and undesired biodistribution. Here we present a simple and clinically relevant method of nanodecoy delivery via inhalation using a nebulizer instead of traditional intravenous injection (Fig. 4a). Inhalation of nanodecoys resulted in the direct accumulation of the therapeutic particles in the lungs, which is one of the primary sites of SARS-CoV-2 infection and replication. From just one single inhalation treatment, DiD-labelled nanodecoys can still be found in the lungs after $72 \mathrm{~h}$ (Fig. 4b). We also detected nanodecoys in the liver, kidney and spleen throughout the $72 \mathrm{~h}$, which can be attributed to the metabolization of the nanodecoys, potentially by macrophages. However, additional studies will be required to elucidate the exact clearance mechanism of the nanodecoys. Many factors, such as particle size, particle characteristics, breathing pattern and treatment duration, can affect the particles' biodistribution and which mechanism is utilized for clearance. For instance, particles deposited in the upper airways are often cleared by mucociliary transport; on the other hand, particles that penetrate to the non-ciliated respiratory epithelium are often cleared or transported via the alveolar macrophages or enter the bloodstream through the alveolar-capillary barrier. The nanodecoys are approximately $300 \mathrm{~nm}(0.3 \mu \mathrm{m})$ in size, and previous reports have shown that similar nanosized particles administered via intravenous injection into the bloodstream are readily cleared by the reticuloendothelial system, in which macrophages also play an important role ${ }^{25,26}$. The ability of the nanodecoys to be recognized and captured by macrophages would contribute to the clearance of SARS-CoV-2 from circulation. Furthermore, we demonstrated that inhalation of nanodecoys could accelerate the removal of SARS-CoV-2 mimics from lungs of infected mice. Mounting evidence indicates that there are increases in inflammatory cytokines in patients with COVID-19, suggesting the existence of a cytokine storm in critically ill patients. It has also been demonstrated that peripheral inflammatory monocytes and pathogenic T cells may induce cytokine storms in patients with severe COVID-19 $9^{27-29}$. Thus, excessive macrophage infiltration may cause undesirable effects.

Recently, nanotechnological tools have been used for the treatment of COVID-19 $9^{30,31}$ and some recent perspectives and research papers hint at the potential of 'nanodecoys' or 'nanosponges' for treating SARS-CoV-2, offering some pilot in vitro or in vivo data. For example, Zhang et al. reported cellular nanosponges comprising everlasting human lung epithelial type II cell-membrane-coated poly(lactic-co-glycolic acid) nanoparticles as a potential countermeasure to SARS-CoV-2, and demonstrated in vitro viral neutralization ${ }^{21}$. Rao et al. fused cell membrane nanovesicles derived from genetically engineered cells (which stably express SARS-CoV-2 receptor ACE2) and human monocytes (which display abundant cytokine receptors for adsorbing viruses and inflammatory cytokines) and tested them in a mouse model of LPS-induced acute lung injury ${ }^{32}$.

\section{Conclusions}

We have provided evidence in a non-human primate model of live SARS-CoV-2 infection that cell-derived and cell-mimicking nanodecoys can protect lung cells from the infection and damage caused by SARS-CoV-2. The cynomolgus macaque model recapitulates many clinical features of human patients with COVID-19. Four doses of nanodecoy inhalation led to a reduction of viral load in both BAL and NS 8 days following SARS-CoV-2 challenge. Adverse events such as weight loss, fever or mortality were not observed. Histopathology, immunohistochemistry, RNAscope and immunofluorescence analyses of lung tissues demonstrated that the nanodecoys were not only effective in alleviating inflammatory cell infiltration and decreasing pulmonary fibrosis but, more importantly, were capable of reducing the levels of SARS-N and vRNA. Our results therefore suggest that LSC-nanodecoys can serve as a potential therapeutic agent for treating COVID-19.

\section{Online content}

Any methods, additional references, Nature Research reporting summaries, source data, extended data, supplementary infor- 
mation, acknowledgements, peer review information; details of author contributions and competing interests; and statements of data and code availability are available at https://doi.org/10.1038/ s41565-021-00923-2.

Received: 23 June 2020; Accepted: 30 April 2021;

Published online: 17 June 2021

\section{References}

1. Wang, C., Horby, P. W., Hayden, F. G. \& Gao, G. F. A novel coronavirus outbreak of global health concern. Lancet 395, 470-473 (2020).

2. Wang, D. et al. Clinical characteristics of 138 hospitalized patients with 2019 novel coronavirus-infected pneumonia in Wuhan, China. JAMA 323, 1061-1069 (2020).

3. Zhao, S. et al. Preliminary estimation of the basic reproduction number of novel coronavirus (2019-nCoV) in China, from 2019 to 2020: a data-driven analysis in the early phase of the outbreak. Int. J. Infect. Dis. 92, 214-217 (2020).

4. Yuan, J., Li, M., Lv, G. \& Lu, Z. K. Monitoring transmissibility and mortality of COVID-19 in Europe. Int. J. Infect. Dis. 95, 311-315 (2020).

5. Ziegler, C. G. K. et al. SARS-CoV-2 receptor ACE2 is an interferon-stimulated gene in human airway epithelial cells and is detected in specific cell subsets across tissues. Cell 181, 1016-1035.e19 (2020).

6. Henry, E. et al. Adult lung spheroid cells contain progenitor cells and mediate regeneration in rodents with bleomycin-induced pulmonary fibrosis. Stem Cells Transl. Med. 4, 1265-1274 (2015).

7. Dinh, P. C. et al. Derivation of therapeutic lung spheroid cells from minimally invasive transbronchial pulmonary biopsies. Respir. Res. 18, 132 (2017).

8. Cores, J. et al. Safety and efficacy of allogeneic lung spheroid cells in a mismatched rat model of pulmonary fibrosis. Stem Cells Transl. Med. 6, 1905-1916 (2017).

9. Dinh, P.-U. C. et al. Inhalation of lung spheroid cell secretome and exosomes promotes lung repair in pulmonary fibrosis. Nat. Commun. 11, 1064 (2020).

10. Jiang, S., Zhang, X. \& Du, L. Therapeutic antibodies and fusion inhibitors targeting the spike protein of SARS-CoV-2. Expert Opin. Ther. Targets 17, 1-7 (2020).

11. Li, Q. et al. The impact of mutations in SARS-CoV-2 spike on viral infectivity and antigenicity. Cell 182, 1284-1294.e1289 (2020).

12. Zhang, L. et al. SARS-CoV-2 spike-protein D614G mutation increases virion spike density and infectivity. Nat. Commun. 11, 6013 (2020).

13. Cores, J. et al. A pre-investigational new drug study of lung spheroid cell therapy for treating pulmonary fibrosis. Stem Cells Transl. Med. 9, 786-798 (2020).

14. Hamming, I. et al. Tissue distribution of ACE2 protein, the functional receptor for SARS coronavirus. A first step in understanding SARS pathogenesis. J. Pathol. 203, 631-637 (2004).
15. Zhang, H., Penninger, J. M., Li, Y., Zhong, N. \& Slutsky, A. S. Angiotensin-converting enzyme 2 (ACE2) as a SARS-CoV-2 receptor: molecular mechanisms and potential therapeutic target. Intensive Care Med. 46, 586-590 (2020).

16. Lan, J. et al. Structure of the SARS-CoV-2 spike receptor-binding domain bound to the ACE2 receptor. Nature 581, 215-220 (2020).

17. Lei, C. et al. Neutralization of SARS-CoV-2 spike pseudotyped virus by recombinant ACE2-Ig. Nat. Commun. 11, 2070 (2020).

18. Kruse, R. L. Therapeutic strategies in an outbreak scenario to treat the novel coronavirus originating in Wuhan, China. F1000Research 9, 72 (2020).

19. Fang, R. H., Luk, B. T., Hu, C.-M. J. \& Zhang, L. Engineered nanoparticles mimicking cell membranes for toxin neutralization. Adv. Drug Deliv. Rev. 90, 69-80 (2015).

20. Rao, L., Tian, R. \& Chen, X. Cell-membrane-mimicking nanodecoys against infectious diseases. ACS Nano 14, 2569-2574 (2020).

21. Zhang, Q. et al. Cellular nanosponges inhibit SARS-CoV-2 infectivity. Nano Lett. 20, 5570-5574 (2020).

22. Rao, L. et al. A biomimetic nanodecoy traps Zika virus to prevent viral infection and fetal microcephaly development. Nano Lett. 19, 2215-2222 (2019).

23. Wei, X. et al. T-cell-mimicking nanoparticles can neutralize HIV infectivity. Adv. Mater. 30, e1802233 (2018).

24. Henry, B. D. et al. Engineered liposomes sequester bacterial exotoxins and protect from severe invasive infections in mice. Nat. Biotechnol. 33, 81-88 (2015).

25. Hoegger, M. J. et al. Assessing mucociliary transport of single particles in vivo shows variable speed and preference for the ventral trachea in newborn pigs. Proc. Natl Acad. Sci. USA 111, 2355-2360 (2014).

26. Geiser, M. Update on macrophage clearance of inhaled micro- and nanoparticles. J. Aerosol Med. Pulm. Drug Deliv. 23, 207-217 (2010).

27. Zhou, Y. et al. Pathogenic T cells and inflammatory monocytes incite inflammatory storm in severe COVID-19 patients. Natl Sci. Rev. 7, 998-1002 (2020)

28. Wang, C. et al. Alveolar macrophage dysfunction and cytokine storm in the pathogenesis of two severe COVID-19 patients. EBioMedicine 57, 102833 (2020).

29. Guo, C. et al. Single-cell analysis of two severe COVID-19 patients reveals a monocyte-associated and tocilizumab-responding cytokine storm. Nat. Commun. 11, 3924 (2020).

30. Florindo, H. F. et al. Immune-mediated approaches against COVID-19. Nat. Nanotechnol. 15, 630-645 (2020).

31. Shin, M. D. et al. COVID-19 vaccine development and a potential nanomaterial path forward. Nat. Nanotechnol. 15, 646-655 (2020).

32. Rao, L. et al. Decoy nanoparticles protect against COVID-19 by concurrently adsorbing viruses and inflammatory cytokines. Proc. Natl Acad. Sci. USA 117, 27141-27147 (2020)

Publisher's note Springer Nature remains neutral with regard to jurisdictional claims in published maps and institutional affiliations.

(c) The Author(s), under exclusive licence to Springer Nature Limited 2021 


\section{Methods}

Generation of nanodecoys. Nanodecoys were derived from LSCs or HEK293 cells (ATCC CRL-1573) by an extruder (LiposoFast LF-50, AVESTIN). Cells were collected and suspended in PBS at a concentration of $5 \times 10^{6} \mathrm{cells} \mathrm{ml}^{-1}$. A large volume of cells could be extruded immediately or stored at $-80^{\circ} \mathrm{C}$ until ready. The cells were passed through the extruder twice through $5 \mu \mathrm{m}, 1 \mu \mathrm{m}$ and $400 \mathrm{~nm}$ pore-sized polycarbonate membrane filters (Avanti Polar Lipids) sequentially. The resulting nanodecoys were purified and concentrated using an ultrafiltration centrifuge tube ( $100 \mathrm{kDa} M_{\mathrm{w}}$ cut-off; Millipore) and centrifuged at $4,500 \mathrm{~g}$ for $10 \mathrm{~min}$ and washed with PBS. The size and concentration of nanodecoys were measured using an nanoparticle tracking analysis system (NanoSight, Malvern Panalytical). Nanodecoys were stored at $4{ }^{\circ} \mathrm{C}$ for 1 week or placed in long-term storage at $-80^{\circ} \mathrm{C}$. The ACE2 receptors on the nanodecoys were detected using immunoblot, immunostaining, flow cytometry and TEM with immunogold labelling.

ACE2 analysis using ELISA. LSC and HEK293 cells $\left(5 \times 10^{6}\right.$ of each) were collected and $10^{10}$ of LSC-nanodecoys and HEK293-nanodecoys were prepared. They were analysed with an ACE2 ELISA kit (ab235649, Abcam) according to the manufacturer's instructions.

In vitro internalization experiments of nanodecoys. Human macrophage primary cells and LSCs $\left(10^{4}\right.$ cells $\left.\mathrm{ml}^{-1}\right)$ were seeded in four-well culture chamber slides (Thermo Fisher Scientific). Nanodecoys $\left(1 \times 10^{6} \mathrm{cells} \mathrm{ml}^{-1}\right)$ were then labelled with DiD and incubated with macrophages or LSCs alone, and also with a co-culture of both (1:1) to mimic the in vivo microenvironment. After $4 \mathrm{~h}$ of incubation, free nanodecoys were removed by three washes with $1 \times$ PBS. Cells were fixed with $4 \%$ paraformaldehyde (PFA) before immunocytochemistry staining with markers for macrophage (CD4; 12-0041-82, Invitrogen) and LSC (CD90; 11-0909-42, Invitrogen) and imaged with an Olympus FluoView confocal microscope. In addition, to quantify the internalization rate of nanodecoys by the different cell types, cells and nanodecoys were cultured in a T75 flask as previously described and collected for flow cytometry analysis (CytoFlex, Beckman Coulter).

In vitro spike $\mathrm{S} 1$ neutralization experiments of nanodecoys. Recombinant spike S1 (40591-V08H, Sino Biological; $10 \mathrm{ng} \mathrm{ml}^{-1}, M_{\mathrm{w}} 76.5 \mathrm{kDa}$ ) was added to nanodecoys at different concentrations $\left(5 \times 10^{9}, 1 \times 10^{9}, 2 \times 10^{8}, 4 \times 10^{7}, 8 \times 10^{6}\right.$, $1.6 \times 10^{6}$ and $3.2 \times 10^{5}$ ) and incubated for $3 \mathrm{~h}$. After that, the unbound spike S1 was removed by ultracentrifugation $(100 \mathrm{kDa})$. Spike $\mathrm{S} 1$ before and after binding to nanodecoys was determined with an ELISA kit (SARS-CoV-2 Spike ELISA Kit, Sino Biological) according to the manufacturer's protocol. To study the neutralization of spike S1 with nanodecoys in primary lung-derived cells (LSCs), spike S1 was first labelled with NHS-Rhodamine (46406, Thermo Fisher Scientific) according to the manufacturer's instructions. The rhodamine $\mathrm{B}(\mathrm{RhB})$-spike S1 $(100 \mathrm{ng})$ was first incubated with LSCs $\left(2 \times 10^{4}\right)$ in four-well slides for $1 \mathrm{~h}$ and washed with PBS three times. DiD-labelled nanodecoys $\left(2 \times 10^{7}\right)$ were then added and incubated for another $4 \mathrm{~h}$. Cells were washed and fixed using $4 \%$ PFA before staining with Alexa Fluor 488 phalloidin (A12379, Invitrogen). Cells were imaged using an Olympus FluoView confocal microscope.

Generation of SARS-CoV-2-mimicking virus. Spike S1 (40591-V08H, Sino Biological) was conjugated to lentivirus (Cellomics Technology) to create a SARS-CoV-2 mimic. The His-tagged spike S1 was linked to Ni-NTA through chemical interaction. NTA with a mercapto group $\left(N-\left[N_{\alpha}, N_{\alpha}\right.\right.$-bis(carboxymethyl)-I -lysine]-12-mercaptododecanamide) was first reacted with 4 -( $N$-maleimidomethyl) cyclohexane-1-carboxylic acid 3-sulfo- $N$-hydroxysuccinimide ester sodium salt (sulfo-SMCC) to give NTA-SMCC and was then added to the lentivirus. The NTA groups were conjugated to the lentivirus through the $-\mathrm{NH}_{2}$ group on the lentivirus and the $N$-hydroxysuccinimide ester on NTA-SMCC. The free NTA-SMCC was removed by centrifugation using an ultrafiltration tube $(100 \mathrm{kDa}$ $M_{\mathrm{w}}$ cut-off; Millipore) to give SARS-CoV-2-mimicking virus (spike S1-lentivirus). The successful conjugation of spike S1 on lentivirus was confirmed using TEM. Briefly, SARS-CoV-2 mimics were incubated with anti-spike S1 antibodies overnight at $4{ }^{\circ} \mathrm{C}$. Free antibodies were removed using an ultrafiltration tube ( $100 \mathrm{kDa} M_{\mathrm{w}}$ cut-off; Millipore) and washed with PBS three times. The spike S1 on the SARS-CoV-2 mimics was labelled with immunogold $(10 \mathrm{~nm})$ antibodies and negatively stained for TEM visualization. The conjugation efficiency of spike S1 on lentivirus was determined using ELISA (SARS-COV-2 Spike ELISA Kit, Sino Biological) according to the manufacturer's protocol.

SARS-CoV-2-mimicking virus in cells. LSC cells $\left(10^{4}\right.$ cells $\left.\mathrm{ml}^{-1}\right)$ were seeded in eight-well culture chamber slides (Thermo Fisher Scientific) and allowed to adhere for $24 \mathrm{~h}$. SARS-CoV-2 mimics $\left(10^{4} \mathrm{TU} \mathrm{ml}^{-1}\right)$ were added into the eight-well slides and incubated for $4 \mathrm{~h}$. Next, LSC cells were washed with PBS twice to remove non-internalized SARS-CoV-2 mimics and stained with $100 \mu \mathrm{M}$ Lyso Dye (Invitrogen, green) at $37^{\circ} \mathrm{C}$ for $30 \mathrm{~min}$. Subsequently, slides were mounted with ProLong Gold Antifade Mountant with 4',6-diamidino-2-phenylindole (DAPI; Invitrogen) and imaged on an Olympus FluoView FV3000 confocal laser scanning microscope with an Olympus UPlanSAPO ×60 objective (1-U2B832).
SARS-CoV-2 mimic neutralization experiment. Nanodecoys were first labelled with DiI. Next, $200 \mu \mathrm{l}$ of SARS-CoV-2 mimic $\left(5 \times 10^{5}\right)$ in $\mathrm{pH} 9.6$ coating buffer was added to each well of 96 -well plates and incubated at $4{ }^{\circ} \mathrm{C}$ overnight for coating. In addition, lentiviruses without spike $\mathrm{S} 1$ were also coated to the plates as a control. Following the incubation, the protein solution was removed, and the wells were washed with $1 \times$ PBS. To study binding, plates were incubated with DiI-labelled nanodecoys at concentrations of $1 \times 10^{4}, 2 \times 10^{4}, 4 \times 10^{4}, 8 \times 10^{4}, 1.6 \times 10^{5}, 3.2 \times 10^{5}$, $6.4 \times 10^{5}$ and $1.28 \times 10^{6}$ for $2 \mathrm{~h}$ at room temperature. The plates were then rinsed with $1 \times$ PBS three times, and fluorescent intensities were determined with a microplate reader (Molecular Devices).

Interaction of SARS-CoV-2 mimic with LSCs was assessed by ICC and flow cytometry. RhB-NHS was first reactivated with NTA-tagged lentivirus and then modified with S1 protein to synthesize RhB-labelled SARS-CoV-2 mimics. LSCs $\left(10^{4}\right.$ cells $\left.\mathrm{ml}^{-1}\right)$ were seeded in four-well culture chamber slides. RhB-labelled lentivirus $\left(10^{4} \mathrm{TU} \mathrm{ml}^{-1}\right)$, RhB-labelled SARS-CoV-2 mimic $\left(10^{4} \mathrm{TU} \mathrm{ml}^{-1}\right)$, RhB-labelled SARS-CoV-2 mimic $\left(10^{4} \mathrm{TU} \mathrm{m}^{-1}\right)+$ LSC-nanodecoys $\left(10^{5}\right)$, and RhB-labelled SARS-CoV-2 mimic $\left(10^{4} \mathrm{TU} \mathrm{ml}^{-1}\right)+$ HEK-nanodecoys $\left(10^{5}\right)$ were incubated with LSCs, respectively. After $4 \mathrm{~h}$ of incubation, free SARS-CoV-2 mimics were removed and washed with PBS three times. Cells were fixed with $4 \%$ PFA, stained for LSC markers (fluorescein isothiocyanate (FITC)-labelled CD90; 11-0909-42, Invitrogen), and imaged with an Olympus FluoView confocal laser scanning microscope. The internalization of SARS-CoV-2 mimics by cells was examined by flow cytometry analysis (CytoFlex, Beckman Coulter).

Nanodecoys protect lung cells from SARS-CoV-2-mimicking viruses. We studied whether nanodecoys could neutralize SARS-CoV-2 mimic viruses and protect lung cells from being infected. Macrophages and LSCs (1:1) were co-cultured in four-well culture chamber slides, and RhB-labelled lentivirus spike $\left(10^{4} \mathrm{TU} \mathrm{mL}^{-1}\right)$ and DiD-labelled nanodecoys $\left(10^{5}\right)$ were added. After $2 \mathrm{~h}$ of incubation, free RhB-labelled lentivirus spike and DiD-labelled nanodecoys were removed and the samples were washed with PBS three times. Cells were fixed with $4 \%$ PFA, stained with LSC (FITC-labelled CD90; 11-0909-42, Invitrogen) or macrophages (CD4) markers, and imaged with an Olympus FluoView confocal laser scanning microscope. Flow cytometry analysis was performed to confirm the microscopy data.

Biodistribution of nanodecoys in mice. All animal procedures were approved by the Institute Animal Care and Use Committee (IACUC) of North Carolina State University (protocol number 19-806-B). Male CD1 mice (aged 7 weeks) were obtained from Charles River Laboratory. DiD-labelled nanodecoys $\left(1 \times 10^{10}\right.$ particles per $\mathrm{kg}$ of body weight) were delivered to the CD1 mice via inhalation treatment using a nebulizer (Pari Trek S Portable Compressor Nebulizer Aerosol System, 047F45-LCS). Mice were killed at 24, 48 and $72 \mathrm{~h}$. All major organs were collected and cryosectioned for further immunofluorescence analysis of the nanodecoys' in vivo biodistribution post-inhalation.

In vivo clearance of the SARS-CoV-2-mimicking virus by nanodecoys in mice. Before performing the clearance assay, the ACE2 level on the nanodecoys was quantified by ELISA (ab235649, Abcam) and determined to be 112 ACE2 per nanodecoy. AF647-labelled SARS-CoV- 2 mimics $\left(5 \times 10^{6}\right.$ per $\mathrm{kg}$ of body weight) were first delivered to the male CD1 mice (aged 7 weeks) via inhalation treatment using a nebulizer (Pari Trek S Portable Compressor Nebulizer Aerosol System, 047F45-LCS). Twenty-four hours later, nanodecoys $\left(1 \times 10^{10}\right.$ particles per kg of body weight) or free rACE2 with the same amount of ACE2 on the nanodecoys were inhaled, respectively. PBS treatment was used as control. Lungs were collected and imaged 1, 2, 3, 4, 5 and $6 \mathrm{~d}$ after treatment using Xenogen Live Imager (IVIS). Additionally, lung tissues were cryosectioned for further analysis of the biodistribution of SARS-CoV-2 mimics in vivo post-inhalation. Blood samples were collected for cytokine array analysis (Mouse Cytokine Array C1000, Raybiotech) according to the manufacturer's instructions.

Toxicity studies in mice. Male CD1 mice (aged 7 weeks) were treated with PBS and LSC- or HEK-nanodecoys $\left(1 \times 10^{10}\right.$ particles per $\mathrm{kg}$ of body weight $)$ via inhalation. After a $14 \mathrm{~d}$ treatment, the blood (blood test) and major organs (H\&E) were collected for toxicity evaluation.

Non-human primate studies. All animal studies were conducted in compliance with all relevant local, state and federal regulations and were approved by the Bioqual IACUC (number 20-090P). Six cynomolgus macaques (three females, three males) were allocated by a counterbalanced randomization. All animals were housed at Bioqual. The macaques were challenged with SARS-CoV-2 using the intranasal and intratracheal routes. The viral inoculum $(0.5 \mathrm{ml})$ was administered dropwise into each nostril and $1.0 \mathrm{ml}$ of viral inoculum was delivered intratracheally using a French rubber catheter/feeding tube, size 10, sterile (cut 4-6 inches in length). Macaques were inoculated with a total dose of $1.1 \times 10^{5}$ p.f.u. SARS-CoV-2. PBS or the LSC-nanodecoys were administered by inhalation using a nebulizer and fitted mask daily from days 2 to 5 following challenge. BAL, NSs, blood, body weight and body temperature were monitored or collected throughout the study. Macaques were necropsied on day 8 post-challenge. All immunologic and virologic assays were performed blinded. 
Statistics and reproducibility. All experiments were performed at least three times independently. Results are shown as means $\pm \mathrm{SD}$. Comparisons between any two groups were performed using a two-tailed, unpaired Student's $t$ test. For multiple group comparison, one-way or two-way analysis of variance (ANOVA) was used with Bonferroni or Tukey post-correction. A $P$ value less than 0.05 was considered statistically significant.

Reporting Summary. Further information on research design is available in the Nature Research Reporting Summary linked to this article.

\section{Data availability}

The data that support the findings of this study are available from the corresponding author upon reasonable request. Source data are provided with this paper.

\section{Acknowledgements}

This work was supported by grants from the National Institutes of Health (HL123920, HL137093, HL144002, HL146153, HL147357 and HL149940 to K.C.) and the American Heart Association (18TPA34230092 and 19EIA34660286 to K.C.). We thank the

Analytical Instrumentation Facility at North Carolina State University for TEM analysis (supported by the State of North Carolina and the National Science Foundation ECCS1542015 and DMR-1726294)

\section{Author contributions}

Z.L. and K.C. conceived and designed the study. Z.L., Z.W. and P.-U.C.D. performed the in vitro and in vivo experiments, interpreted the results and wrote the paper. K.D.P. and H.L. helped with the ICC and Western blot experiments. D.Z. and S.H. helped with the flow cytometry experiments. M.G.L., A.C., H.A., J.G. and L.P. performed the non-human primate studies. L.J.L. helped with the writing of the paper.

\section{Competing interests}

K.C. is a co-founder and equity holder of BreStem Therapeutics Inc. P.-U.C.D. reports compensation for consulting from BreStem Therapeutics Inc. The remaining authors declare no competing interests.

\section{Additional information}

Supplementary information The online version contains supplementary material available at https://doi.org/10.1038/s41565-021-00923-2.

Correspondence and requests for materials should be addressed to K.C.

Peer review information Nature Nanotechnology thanks Emma Chory and Andreas Herrmann for their contribution to the peer review of this work

Reprints and permissions information is available at www.nature.com/reprints. 


\section{Reporting Summary}

Nature Research wishes to improve the reproducibility of the work that we publish. This form provides structure for consistency and transparency in reporting. For further information on Nature Research policies, see our Editorial Policies and the Editorial Policy Checklist.

\section{Statistics}

For all statistical analyses, confirm that the following items are present in the figure legend, table legend, main text, or Methods section.

n/a Confirmed

$\square$ The exact sample size $(n)$ for each experimental group/condition, given as a discrete number and unit of measurement

$\square$ \ A statement on whether measurements were taken from distinct samples or whether the same sample was measured repeatedly

$\square$ The statistical test(s) used AND whether they are one- or two-sided

$\square$ Only common tests should be described solely by name; describe more complex techniques in the Methods section.

Х $\square$ A description of all covariates tested

$\square$ \ A description of any assumptions or corrections, such as tests of normality and adjustment for multiple comparisons

$\square$ A full description of the statistical parameters including central tendency (e.g. means) or other basic estimates (e.g. regression coefficient)

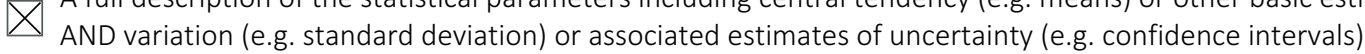

$\varnothing$ For null hypothesis testing, the test statistic (e.g. $F, t, r$ ) with confidence intervals, effect sizes, degrees of freedom and $P$ value noted

Give P values as exact values whenever suitable.

Х $\square$ For Bayesian analysis, information on the choice of priors and Markov chain Monte Carlo settings

Х $\square$ For hierarchical and complex designs, identification of the appropriate level for tests and full reporting of outcomes

\ $\square$ Estimates of effect sizes (e.g. Cohen's $d$, Pearson's $r$ ), indicating how they were calculated

Our web collection on statistics for biologists contains articles on many of the points above.

\section{Software and code}

Policy information about availability of computer code

Data collection Flow Cytometry analysis was collected on the CytoFlex (Beckman Coulter) and LSR-II (BD). Exosome particle analysis was collected on the Nanoparticle Tracking Analysis (NTA; NanoSight).

Electron microscopy images were collected on the JEOL JEM-2000FX.

Fluorescent imaging was collected on the Olympus FLUOVIEW confocal microscope, FV31S-SW viewer version 2.4 (Olympus).

Western blot images were collected on the BioRad ChemiDoc Imaging System.

ELISAs analysis was collected on the Synergy neo2 multi-mode reader (BioTeK).

In vivo and ex vivo fluorescent imaging was collected on Xenogen Live Imager (IVIS).

RT-PCR analysis was collected on the QuantStudio 6 and 7 Flex Real Time PCR System (Applied Biosystems)

Data analysis Histological staining images acquired with FV-31S-SW software and were analyzed by Image J version 1.8.0 to generate statistical data. Flow cytometry analysis was performed on FCS Express 6 (De Novo Software) and FACSDiva Software (BD). All the statistical data were analyzed using GraphPad Prism version 8.0.

For manuscripts utilizing custom algorithms or software that are central to the research but not yet described in published literature, software must be made available to editors and reviewers. We strongly encourage code deposition in a community repository (e.g. GitHub). See the Nature Research guidelines for submitting code \& software for further information. 
Policy information about availability of data

All manuscripts must include a data availability statement. This statement should provide the following information, where applicable:

- Accession codes, unique identifiers, or web links for publicly available datasets

- A list of figures that have associated raw data

- A description of any restrictions on data availability

Raw data from this study is available in the Source Data File. Additionally, all data from this study is available from the corresponding author upon reasonable request.

\section{Field-specific reporting}

Please select the one below that is the best fit for your research. If you are not sure, read the appropriate sections before making your selection. \Life sciences Behavioural \& social sciences Ecological, evolutionary \& environmental sciences

For a reference copy of the document with all sections, see nature.com/documents/nr-reporting-summary-flat.pdf

\section{Life sciences study design}

All studies must disclose on these points even when the disclosure is negative.

Sample size All experiments were performed at least in triplicate independent measurements or more as indicated in the text. Sample size for in-vivo experiment was determined by power analysis using statistics from previous experiments and preliminary experiments. Six cynomolgus macaques (three per group) were used in the live SARS-CoV-2 challenge experiments.

Data exclusions No data was excluded.

Replication All experiments were performed at least in triplicates, and all attempts at replication were successful.

Randomization All samples and animals grouping were randomly assigned. The NHPs study utilized a counterbalanced randomization to account for age and sex distribution.

Blinding Data acquisition and analysis were performed by investigators who are blinded to the group allocation.

\section{Behavioural \& social sciences study design}

All studies must disclose on these points even when the disclosure is negative.

Study description

Research sample

Sampling strategy

Data collection

Timing

Data exclusions

Non-participation

Randomization
Briefly describe the study type including whether data are quantitative, qualitative, or mixed-methods (e.g. qualitative cross-sectional, quantitative experimental, mixed-methods case study).

State the research sample (e.g. Harvard university undergraduates, villagers in rural India) and provide relevant demographic information (e.g. age, sex) and indicate whether the sample is representative. Provide a rationale for the study sample chosen. For studies involving existing datasets, please describe the dataset and source.

Describe the sampling procedure (e.g. random, snowball, stratified, convenience). Describe the statistical methods that were used to predetermine sample size OR if no sample-size calculation was performed, describe how sample sizes were chosen and provide a rationale for why these sample sizes are sufficient. For qualitative data, please indicate whether data saturation was considered, and what criteria were used to decide that no further sampling was needed.

Provide details about the data collection procedure, including the instruments or devices used to record the data (e.g. pen and paper, computer, eye tracker, video or audio equipment) whether anyone was present besides the participant(s) and the researcher, and whether the researcher was blind to experimental condition and/or the study hypothesis during data collection.

Indicate the start and stop dates of data collection. If there is a gap between collection periods, state the dates for each sample cohort.

If no data were excluded from the analyses, state so OR if data were excluded, provide the exact number of exclusions and the rationale behind them, indicating whether exclusion criteria were pre-established.

State how many participants dropped out/declined participation and the reason(s) given OR provide response rate OR state that no participants dropped out/declined participation.

If participants were not allocated into experimental groups, state so OR describe how participants were allocated to groups, and if allocation was not random, describe how covariates were controlled. 


\section{Ecological, evolutionary \& environmental sciences study design}

All studies must disclose on these points even when the disclosure is negative.

Study description

Research sample

Sampling strategy

Data collection

Timing and spatial scale

(n) Indicate the start and stop dates of data collection, noting the frequency and periodicity of sampling and providing a rationale for these choices. If there is a gap between collection periods, state the dates for each sample cohort. Specify the spatial scale from which the data are taken

Data exclusions

If no data were excluded from the analyses, state so OR if data were excluded, describe the exclusions and the rationale behind them, indicating whether exclusion criteria were pre-established.

Reproducibility

Describe the measures taken to verify the reproducibility of experimental findings. For each experiment, note whether any attempts to repeat the experiment failed OR state that all attempts to repeat the experiment were successful.

Randomization

Describe how samples/organisms/participants were allocated into groups. If allocation was not random, describe how covariates were controlled. If this is not relevant to your study, explain why.

Blinding

Describe the extent of blinding used during data acquisition and analysis. If blinding was not possible, describe why OR explain why blinding was not relevant to your study.

Did the study involve field work? $\square$ Yes $\square$ No

Field work, collection and transport

Field conditions

Describe the study conditions for field work, providing relevant parameters (e.g. temperature, rainfall).

Location

State the location of the sampling or experiment, providing relevant parameters (e.g. latitude and longitude, elevation, water depth).

Access \& import/export

Describe the efforts you have made to access habitats and to collect and import/export your samples in a responsible manner and in compliance with local, national and international laws, noting any permits that were obtained (give the name of the issuing authority, the date of issue, and any identifying information).

Disturbance

\section{Reporting for specific materials, systems and methods}

We require information from authors about some types of materials, experimental systems and methods used in many studies. Here, indicate whether each material, system or method listed is relevant to your study. If you are not sure if a list item applies to your research, read the appropriate section before selecting a response.

Materials \& experimental systems

\begin{tabular}{l|l}
\hline$n / a$ & Involved in the study \\
$\square$ Antibodies \\
$\square$
\end{tabular}

Methods

\begin{tabular}{l|l}
\hline n/a & Involved in the study \\
$\square$ & $\square$ ChIP-seq \\
$\square$ & $\bigotimes$ Flow cytometry \\
$\searrow$ & $\square$ MRI-based neuroimaging
\end{tabular}



Invitrogen), CD90 (11-0909-42, Invitrogen), CD68 (ab955; Abcam), EpCAM (ab71916; Abcam), CD90 (555595; BD), MUC5b (ab77995; Abcam), VWF (ab11713; Abcam), Primary rabbit anti-SARS-N antibody (NB100-56576, Novus), goat anti-rabbit HRP secondary antibody (ab6721, Abcam), goat anti-rabbit Alexa Fluor 594 (ab150080, Abcam), AF-488-CD206 (sc-376108, Santa Cruz Biotechnologies), efluor 660-CD68 (50-0681-82, eBioscience), Alexa Fluor ${ }^{\circledR}$ 568-Iba-1 (ab221003, Abcam), and the FITC-pan-CK ( ab78478, abcam)

Validation

All antibodies are commercially available. Antibodies were validated for species and applications, as appropriate, accordingly to the manufacturer's specification sheet and supported by relevant citations on their product pages. Appropriate controls were used for all flow cytometry, immunoblotting and immunostaining.

\section{Eukaryotic cell lines}

Policy information about cell lines

Cell line source(s)

HEK293 (ATCC ${ }^{\circledR}$ CRL-1573 ${ }^{\text {TM }}$ ) and Human lung fibroblast cells (ATCC ${ }^{\circledR}$ PCS-201-013 ${ }^{\text {TM }}$ ) were purchased from ATCC. Human macrophage primary cells (36070-01) were purchased from CELPROGEN. Lung spheroid cells (LSC) and explant derived cells (EDC) were generated from healthy human whole lung samples from the Cystic Fibrosis and Pulmonary Diseases Research and Treatment Center at the University of North Carolina at Chapel Hill with appropriate IRB approval and informed consent.

Authentication

Human macrophage primary cells were authenticated by the expression of macrophage markers. Lung spheroid cells were authenticated by our previous reported surface markers (Stem Cells Transl. Med. 4, 1265-1274, 2015).

Mycoplasma contamination Mycoplasma testing (PCR method) is routinely performed in the lab, and all cell lines were tested negative for mycoplasma contamination.

Commonly misidentified lines (See ICLAC register)

No misidentified cell lines were used in the study.

\section{Palaeontology and Archaeology}

Specimen provenance Provide provenance information for specimens and describe permits that were obtained for the work (including the name of the issuing authority, the date of issue, and any identifying information).

Specimen deposition Indicate where the specimens have been deposited to permit free access by other researchers.

Dating methods

If new dates are provided, describe how they were obtained (e.g. collection, storage, sample pretreatment and measurement), where they were obtained (i.e. lab name), the calibration program and the protocol for quality assurance OR state that no new dates are provided.

Tick this box to confirm that the raw and calibrated dates are available in the paper or in Supplementary Information.

Ethics oversight

Identify the organization(s) that approved or provided guidance on the study protocol, OR state that no ethical approval or guidance was required and explain why not.

Note that full information on the approval of the study protocol must also be provided in the manuscript.

\section{Animals and other organisms}

Policy information about studies involving animals; ARRIVE guidelines recommended for reporting animal research $\begin{array}{ll}\text { Laboratory animals } & \text { Seven-week-old male CD1 mice (Crl:CD1(ICR)) were obtained from Charles River Laboratory (Wilmington, MA, USA). Six Cynomolgus } \\ \text { Macaques (three females, three males) were purchased and housed at Bioqual, Inc. (Rockville, MD, USA). }\end{array}$

Wild animals No wild animals were used in this study.

Field-collected samples No field-collected samples were used in this study.

Ethics oversight $\quad$ The mice study was performed under guidelines of the North Carolina State University, Institutional Animal Care and Use Committee(IACUC), under approved IACUC \# 19-806-B.

The cynomolgus macaques study was approved by the Bioqual Institutional Animal Care and Use Committee (IACUC) under approved IACUC \# 20-090P.

Note that full information on the approval of the study protocol must also be provided in the manuscript. 
Policy information about studies involving human research participants

Population characteristics

Describe the covariate-relevant population characteristics of the human research participants (e.g. age, gender, genotypic information, past and current diagnosis and treatment categories). If you filled out the behavioural \& social sciences study design questions and have nothing to add here, write "See above."

Recruitment

Describe how participants were recruited. Outline any potential self-selection bias or other biases that may be present and how these are likely to impact results.

Ethics oversight

Identify the organization(s) that approved the study protocol.

Note that full information on the approval of the study protocol must also be provided in the manuscript.

\section{Clinical data}

Policy information about clinical studies

All manuscripts should comply with the ICMJE guidelines for publication of clinical research and a completed CONSORT checklist must be included with all submissions.

Clinical trial registration

Study protocol

Data collection

Outcomes
Provide the trial registration number from ClinicalTrials. gov or an equivalent agency.

Note where the full trial protocol can be accessed OR if not available, explain why.

Describe the settings and locales of data collection, noting the time periods of recruitment and data collection.

Describe how you pre-defined primary and secondary outcome measures and how you assessed these measures.

\section{Dual use research of concern}

Policy information about dual use research of concern

\section{Hazards}

Could the accidental, deliberate or reckless misuse of agents or technologies generated in the work, or the application of information presented in the manuscript, pose a threat to:
No Yes
$\square \square$ Public health
$\square \square$ National security
$\square \square$ Crops and/or livestock
$\square \square$ Ecosystems
$\square \square$ Any other significant area

\section{Experiments of concern}

Does the work involve any of these experiments of concern:
No $\mid$ Yes
$\square \square$ Demonstrate how to render a vaccine ineffective
$\square \square$ Confer resistance to therapeutically useful antibiotics or antiviral agents
$\square \square$ Enhance the virulence of a pathogen or render a nonpathogen virulent
$\square \square$ Increase transmissibility of a pathogen
$\square \square$ Alter the host range of a pathogen
$\square \square$ Enable evasion of diagnostic/detection modalities
$\square \square$ Enable the weaponization of a biological agent or toxin
$\square$ Any other potentially harmful combination of experiments and agents

\section{ChIP-seq}

\section{Data deposition}

$\square$ Confirm that both raw and final processed data have been deposited in a public database such as GEO.

$\square$ Confirm that you have deposited or provided access to graph files (e.g. BED files) for the called peaks. 
Data access links

May remain private before publication

Files in database submission

Genome browser session (e.g. UCSC)
For "Initial submission" or "Revised version" documents, provide reviewer access links. For your "Final submission" document, provide a link to the deposited data.

Provide a list of all files available in the database submission.

Provide a link to an anonymized genome browser session for "Initial submission" and "Revised version" documents only, to enable peer review. Write "no longer applicable" for "Final submission" documents.

\section{Methodology}

Replicates

Sequencing depth

Describe the experimental replicates, specifying number, type and replicate agreement.

Antibodies

Describe the sequencing depth for each experiment, providing the total number of reads, uniquely mapped reads, length of reads and whether they were paired-or single-end.

Peak calling parameters

Describe the antibodies used for the ChIP-seq experiments; as applicable, provide supplier name, catalog number, clone name, and lot number.

Data quality

Software

Specify the command line program and parameters used for read mapping and peak calling, including the ChIP, control and index files used.

Describe the methods used to ensure data quality in full detail, including how many peaks are at FDR 5\% and above 5-fold enrichment.

Describe the software used to collect and analyze the ChIP-seq data. For custom code that has been deposited into a community repository, provide accession details.

\section{Flow Cytometry}

Plots

Confirm that:

\The axis labels state the marker and fluorochrome used (e.g. CD4-FITC).

$\bigotimes$ The axis scales are clearly visible. Include numbers along axes only for bottom left plot of group (a 'group' is an analysis of identical markers).

$\bigotimes$ All plots are contour plots with outliers or pseudocolor plots.

$\bigotimes$ A numerical value for number of cells or percentage (with statistics) is provided.

Methodology

Sample preparation

Cells were washed with MACS flow buffer (130-091-222; MACS) and permeabilized with BD Cytofix/Cytoperm (554714; BD) prior to incubation with antibodies against ACE2 (PA5-85139; Invitrogen), EpCAM (ab71916; Abcam), CD90 (555595; BD), MUC5b (ab77995; Abcam), and VWF (ab11713; Abcam).

Instrument BD LSR-II Flow and CytoFlex (Beckman Coulter)

Software BD FACSDiva Software and FCS Express 6 (De Novo Software)

Cell population abundance Provided in Supplementary Fig. 3 and 4

Gating strategy

Unstained cells were used as negative control and FSC-A/SSC-A was used to gate singlets, followed by setting up a gate that 98\%-100\% unstained cells as negative. The details were provided in Supplementary in Fig. 17and 18.

$\bigotimes$ Tick this box to confirm that a figure exemplifying the gating strategy is provided in the Supplementary Information.

\section{Magnetic resonance imaging}

Experimental design

Design type

Design specifications

Behavioral performance measures

Indicate task or resting state; event-related or block design.

Specify the number of blocks, trials or experimental units per session and/or subject, and specify the length of each trial or block (if trials are blocked) and interval between trials.

State number and/or type of variables recorded (e.g. correct button press, response time) and what statistics were used to establish that the subjects were performing the task as expected (e.g. mean, range, and/or standard deviation across subjects). 
Acquisition

Imaging type(s)

Specify: functional, structural, diffusion, perfusion.

Field strength

Specify in Tesla

Sequence \& imaging parameters

Specify the pulse sequence type (gradient echo, spin echo, etc.), imaging type (EPI, spiral, etc.), field of view, matrix size slice thickness, orientation and TE/TR/flip angle.

Area of acquisition

State whether a whole brain scan was used OR define the area of acquisition, describing how the region was determined.

Diffusion MRI

$\square$ Used

Not used

Preprocessing

Preprocessing software

Provide detail on software version and revision number and on specific parameters (model/functions, brain extraction, segmentation, smoothing kernel size, etc.).

Normalization

If data were normalized/standardized, describe the approach(es): specify linear or non-linear and define image types used for transformation OR indicate that data were not normalized and explain rationale for lack of normalization.

Normalization template

Describe the template used for normalization/transformation, specifying subject space or group standardized space (e.g. original Talairach, MNI305, ICBM152) OR indicate that the data were not normalized.

Noise and artifact removal

Describe your procedure(s) for artifact and structured noise removal, specifying motion parameters, tissue signals and physiological signals (heart rate, respiration).

Volume censoring

Define your software and/or method and criteria for volume censoring, and state the extent of such censoring.

Statistical modeling \& inference

Model type and settings

Specify type (mass univariate, multivariate, RSA, predictive, etc.) and describe essential details of the model at the first and second levels (e.g. fixed, random or mixed effects; drift or auto-correlation).

Effect(s) tested

Define precise effect in terms of the task or stimulus conditions instead of psychological concepts and indicate whether ANOVA or factorial designs were used.

Specify type of analysis:

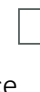

Statistic type for inference (See Eklund et al. 2016)

Correction
Whole brain
ROI-based
Both

Specify voxel-wise or cluster-wise and report all relevant parameters for cluster-wise methods.

Describe the type of correction and how it is obtained for multiple comparisons (e.g. FWE, FDR, permutation or Monte Carlo).

Models \& analysis

$\mathrm{n} / \mathrm{a}$ Involved in the study

$\square \square$ Functional and/or effective connectivity

$\square$ Graph analysis

$\square$ Multivariate modeling or predictive analysis

Functional and/or effective connectivity

Report the measures of dependence used and the model details (e.g. Pearson correlation, partial correlation, mutual information).

Graph analysis

Report the dependent variable and connectivity measure, specifying weighted graph or binarized graph, subject-or group-level, and the global and/or node summaries used (e.g. clustering coefficient, efficiency, etc.).

Multivariate modeling and predictive analysis

Specify independent variables, features extraction and dimension reduction, model, training and evaluation metrics. 